\title{
Expression profiling of spinal cord dorsal horn in a rat model of complex regional pain syndrome type-I uncovers potential mechanisms mediating pain and neuroinflammation responses
}

\author{
Ruixiang Chen ${ }^{1 \dagger}$, Chengyu Yin ${ }^{1 \dagger}$, Qimiao Hu ${ }^{1 \dagger}$, Boyu Liu' ${ }^{1}$, Yan Tai ${ }^{2}$, Xiaoli Zheng ${ }^{1}$, Yuanyuan $\mathrm{Li}^{1}$,
} Jianqiao Fang ${ }^{1 *}$ and Boyi Liu ${ }^{1 *}$ (D)

\begin{abstract}
Background: Complex regional pain syndrome type-I (CRPS-I) is a progressive and devastating pain condition. The mechanisms of CRPS-I still remain poorly understood. We aim to explore expression profiles of genes relevant to pain and neuroinflammation mechanisms involved in CRPS-I.

Methods: The rat chronic post-ischemic pain (CPIP) model that mimics human CRPS-I was established. RNAsequencing (RNA-Seq), qPCR, Western blot, immunostaining, and pharmacological studies were used for profiling gene changes in ipsilateral spinal cord dorsal horn (SCDH) Of CPIP model rat and further validation.

Results: CPIP rats developed persistent mechanical allodynia in bilateral hind paws, accompanied with obvious glial activation in SCDH. RNA-Seq identified a total of 435 differentially expressed genes (DEGs) in ipsilateral SCDH of CPIP rats. QPCR confirmed the expression of several representative genes. Functional analysis of DEGs identified that the most significantly enriched biological processes of upregulated genes include inflammatory and innate immune response. We further identified NLRP3 inflammasome expression to be significantly upregulated in SCDH of CPIP rats. Pharmacological blocking NLRP3 inflammasome reduced IL-1 $\beta$ overproduction, glial activation in SCDH as well as mechanical allodynia of CPIP rats.
\end{abstract}

Conclusion: Our study revealed that immune and inflammatory responses are predominant biological events in SCDH of CPIP rats. We further identified NLRP3 inflammasome in SCDH as a key contributor to the pain and inflammation responses in CPIP rats. Thus, our study provided putative novel targets that may help to develop effective therapeutics against CRPS-I.

Keywords: RNA-Seq, Pain, CRPS-I, Spinal cord dorsal horn, Inflammation, Inflammasome, Cytokine

\footnotetext{
*Correspondence: fangjianqiao7532@163.com; boyi.liu@foxmail.com

${ }^{\dagger}$ Ruixiang Chen, Chengyu Yin and Qimiao Hu contributed equally to this work.

'Department of Neurobiology and Acupuncture Research, The Third Clinical Medical College, Zhejiang Chinese Medical University, Key Laboratory of Acupuncture and Neurology of Zhejiang Province, 548 Binwen Road, Hangzhou 310053, China

Full list of author information is available at the end of the article
}

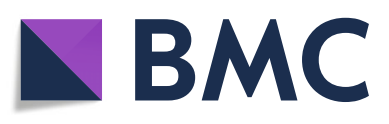

( ) The Author(s). 2020 Open Access This article is licensed under a Creative Commons Attribution 4.0 International License, which permits use, sharing, adaptation, distribution and reproduction in any medium or format, as long as you give appropriate credit to the original author(s) and the source, provide a link to the Creative Commons licence, and indicate if changes were made. The images or other third party material in this article are included in the article's Creative Commons licence, unless indicated otherwise in a credit line to the material. If material is not included in the article's Creative Commons licence and your intended use is not permitted by statutory regulation or exceeds the permitted use, you will need to obtain permission directly from the copyright holder. To view a copy of this licence, visit http://creativecommons.org/licenses/by/4.0/. The Creative Commons Public Domain Dedication waiver (http://creativecommons.org/publicdomain/zero/1.0/) applies to the data made available in this article, unless otherwise stated in a credit line to the data. 


\section{Introduction}

Complex regional pain syndrome type-I (CRPS-I) is a progressive and devastating neuropathic pain condition that usually affects the limb and is not accompanied with a clinically verifiable nerve injury [1]. CRPS-I usually develops after an initial injury, which includes ischemia, soft tissue trauma, surgery, or fractures to the extremity $[2,3]$. CRPS-I can develop into chronic pain state that severely affects the patient's life quality [4]. There are currently no specific drugs approved for the treatment of CRPS. Conventional treatments for CRPS-I include physiotherapy, sympathetic blockade, corticosteroids, and non-steroidal anti-inflammatory drugs (NSAIDs) [5]. However, none of the above treatment options produce satisfactory relieving effects on CRPS-I, which makes it one of the most clinically challenging neuropathic pain conditions [6].

Currently, the mechanisms of CRPS-I are still not fully understood. Ischemia/reperfusion injury is among one of the major causes leading to CRPS-I [7, 8]. In order to get more understandings of the mechanisms of CRPS-I, Coderre et al. established a rat chronic post-ischemic pain (CPIP) model by applying prolonged hind paw ischemia and reperfusion to mimic CRPS-I [9]. The CPIP model displayed many CRPS-I-like symptoms, including early hind paw edema, hyperemia, and skin warmth, accompanied with long-lasting neuropathic pain conditions, including bilateral mechanical and thermal hypersensitivities [9]. With the aid of this animal model, it is proposed that central pain sensitization, reactive oxygen species increase, TRPA1 activation, etc. may contribute to CRPS-I pathology [10-12]. We recently contributed to these efforts by performing transcriptome profiling of the dorsal root ganglia (DRG) of CPIP model rat and identified potential peripheral pain mechanisms involved in CRPS-I [13]. Our recent work further identified the pain-sensing ion channel TRPV1 in the DRG neurons as a key target involved in mediating the pain symptoms of CPIP model rats [14].

The spinal cord dorsal horn $(\mathrm{SCDH})$ receives pain signal inputs from the peripheral sensory neurons and plays a critical role in integrating pain signals and central pain sensitization. Non-neuronal cells, such as astrocytes and microglia, are activated in SCDH of CPIP model rats and produce pro-inflammatory mediators, such as some cytokines and chemokines that can modulate pain process $[11,15,16]$. These substances act on spinal nociceptive neurons to produce neuroinflammation and sensitize pain-related receptors or ion channels to initiate central pain sensitization [17]. In order to further explore the central mechanisms underlying CRPS-I, we proceeded to carry out genome-wide expression profiling of the ipsilateral SCDH of CPIP model rats and sham control rats using RNA-Seq. We identified a number of differentially expressed genes (DEGs). We further examined the molecular/cellular functions and the signaling pathways that these DEGs were involved in. We compared our findings with previously published datasets of neuropathic pain models and identified a core set of genes and pathways that extensively participated in CPIP and other neuropathic pain conditions. By analyzing the RNA-Seq dataset, we further identified NLRP3 inflammasome in $\mathrm{SCDH}$ is a key player in mediating the pain and inflammation responses in CPIP model rats. Our work uncovered the gene expression patterns in the SCDH of a rat model of CRPS-I, which is essential for understanding the neuroinflammation and pain mechanisms of CRPS-I. Our work may provide further insights into identifying novel and effective therapeutic targets against CRPS-I.

\section{Materials and methods \\ Animals}

Male Sprague-Dawley rats (8-10 weeks, 300-320 g) were purchased from Shanghai Laboratory Animal Center, Chinese Academy of Sciences and housed in the Laboratory Animal Center of Zhejiang Chinese Medical University accredited by the Association for Assessment and Accreditation of Laboratory Animal Care (AAALAC) under standard environmental conditions (12-h lightdark cycles and $24 \pm 2{ }^{\circ} \mathrm{C}$ ). Food and water were provided ad libitum. Rats were randomly allocated and 4 rats were housed per cage. The rats were given a minimum of 1 week to adapt to the new environment before the experiment.

\section{CPIP rat model establishment}

CPIP was established via prolonged hind paw ischemia and reperfusion as described previously $[9,18]$. Anesthesia was induced in all rats with an intraperitoneal injection of $50 \mathrm{mg} / \mathrm{kg}$ of sodium phenobarbital and was maintained with an infusion of sodium phenobarbital at $20 \mathrm{mg} / \mathrm{kg} / \mathrm{h}$. An O-ring with 7/32 internal diameter was tightly passed around the right hind limb just proximal to the ankle joint. The O-ring was then cut off $3 \mathrm{~h}$ later for reperfusion. Sham rats received the same anesthetic procedure but the ankle was surrounded with a cut O-ring which did not block blood flow.

\section{Determination of mechanical allodynia}

Rats were habituated to the test environment daily for a consecutive 3 days before baseline test. Rats were individually placed in transparent Plexiglas chambers on an elevated mesh floor and were habituated for $30 \mathrm{~min}$ before the test. The mechanical hyperalgesia was determined using a series of von Frey filaments (UGO Basile, Italy) applied perpendicularly to the midplantar surface of the hind paws, with sufficient force to bend the 
filament slightly for $3-5 \mathrm{~s}$ according to methods we previously used $[19,20]$. An abrupt withdrawal of the paw and licking and vigorously shaking in response to stimulation were considered pain-like responses. The threshold was determined using the up-down testing paradigm, and the $50 \%$ paw withdrawal threshold (PWT) was calculated by the nonparametric Dixon test [21]. Behavior tests were all conducted by an experimenter blinded to experimental conditions.

\section{Determination of the hind paw swelling}

Swelling was observed as an increase in hind paw diameter, as measured by a digital caliper, and was calculated as the difference between the basal value and the test value as in our previous study [14]. Changes in paw diameter were shown as $\%$ increase in paw diameter and calculated as follows: \% increase in paw diameter $=\left(D_{\text {af- }}\right.$ ter $-D_{\text {before }} / D_{\text {before. }}$ Each rat was measured 3 times, and the mean value was calculated.

\section{Tissue collect and RNA extraction}

The L4-6 spinal cord dorsal horn segments ipsilateral to the side of ischemia/reperfusion injury were harvested and used for generating libraries for RNA-seq. At day 7, rats were deeply anesthetized with sodium pentobarbital $(40 \mathrm{mg} / \mathrm{kg})$ and were perfused transcardially with 200 $\mathrm{mL} 0.9 \% \mathrm{NaCl}\left(4^{\circ} \mathrm{C}\right)$. After the perfusion, the L4-6 spinal segments were extracted by lumbar laminectomy, separated into left (contralateral) and right (ipsilateral) cord, and the ipsilateral dorsal horn was immediately preserved in the RNA later solution (Invitrogen, Carlsbad, USA). Total RNA was extracted using Trizol reagent (Invitrogen, Carlsbad, USA) according to the manufacturer's instructions with DNase I to degrade contaminating DNA. The purity and concentration of the samples was assessed by the Nanodrop Spectrophotometer (NanoDrop Products, CA, USA), and the RNA integrity was assessed by the Agilent 2100 Bioanalyzer (Agilent Technologies, Palo Alto, CA).

\section{Immunofluorescence staining}

Rats were deeply anesthetized with sodium pentobarbital $(50 \mathrm{mg} / \mathrm{kg})$ and were perfused transcardially with $200 \mathrm{~mL}$ $0.9 \%$ saline $\left(4^{\circ} \mathrm{C}\right)$ followed by $200 \mathrm{ml}$ of $4 \%$ formaldehyde. The ipsilateral L4-6 spinal cord was harvested (contralateral side of the spinal cord was labeled by piercing a needle into the anterior horn) and post-fixed in the same fixative for $4 \mathrm{~h}\left(4^{\circ} \mathrm{C}\right)$ before transfer to $15 \%$ and $30 \% \mathrm{su}-$ crose for $72 \mathrm{~h}$ for dehydration. Several days later, the spinal cord was serially cut into $25-\mu \mathrm{m}$-thick transverse sections on a frozen microtome (Thermo NX50, USA) and mounted on gelatin-coated glass slides as 6 sets of every 5 th serial sections. All the slides were blocked with $5 \%$ normal donkey serum in TBST (with $0.1 \%$ Tween-20) for $1 \mathrm{~h}$ at $37^{\circ} \mathrm{C}$ and then incubated overnight with corresponding primary antibodies. The primary antibodies used were mouse anti-GFAP (\#c9205, Sigma) and mouse antiOX42 (\#ab1211, Abcam). The following day, the sections were rinsed with TBST $(6 \times 10 \mathrm{~min})$ and incubated for $1 \mathrm{~h}$ with a mixture of corresponding secondary antibodies. Fluorescence images were captured by Nikon A1R laser scanning confocal microscope (Nikon, Japan). For quantitative fluorescence intensity analysis, uniform microscope settings were maintained throughout all image capture sessions. All stained sections were examined and analyzed in a blinded manner. Five images were randomly selected per rat tissue and averaged and then compared according to methods described in our previous studies $[22,23]$.

\section{RNA-Seq library establishment and RNA-Seq}

Total mRNAs from 3 rats of each group were isolated and used to construct sequencing libraries. mRNA molecules were purified from total RNA using oligo (dT)-attached magnetic beads. mRNAs were fragmented into small pieces using fragmentation reagent. First-strand cDNA was generated using random hexamer-primed reverse transcription and then was followed by a secondstrand cDNA synthesis. The synthesized cDNA was subjected to end-repair and then was 3' adenylated. Adaptors were ligated to the ends of these $3^{\prime}$ adenylated cDNA fragments. This process is to amplify the cDNA fragments with adaptors from the previous step. PCR products are purified with the SPRI beads and dissolved in EB solution. The double-stranded PCR products were heat denatured and circularized by the splint oligosequence. The single-strand circle DNA (ssCir DNA) was formatted as the final library. Library was validating on the Agilent Technologies 2100 bioanalyzer. The library was amplified with phi29 to make DNA nanoball (DNB) which have more than 300 copies of one molecular. The DNBs were load into the patterned nanoarray and single end 50 bases reads were generated in the way of sequencing by synthesis. Finally, the fragments were enriched by PCR amplification to construct a library ready for sequencing using BGISEQ-500 by BGI (Shenzhen, China).

\section{Bioinformatics analysis}

Primary sequencing data produced by RNA-Seq (raw reads) were subjected to quality control (QC). The information of total reads and mapping ratio reads were shown in Table 1. Raw data were filtered into clean reads by internal software SOAPnuke (version 1.5.2), as follows: Remove reads in which unknown bases $(\mathrm{N})$ are more than 10\%; Remove reads with adaptors; Remove low-quality reads (we define the low-quality read as the percentage of base which quality is lesser than 15 and greater than $50 \%$ in a read). QC of alignment was 
Table 1 The information of total reads and mapping ratio for Sham and CPIP groups in RNA-Seq

\begin{tabular}{llllll}
\hline Sample & Total raw reads $(\mathrm{Mb})$ & Total clean reads $(\mathrm{Mb})$ & Total clean bases (Gb) & Clean reads Q20 (\%) & Clean reads Q30 (\%) \\
\hline SCDH model 1 & 21.94 & 21.92 & 1.1 & 98.56 & 92.23 \\
SCDH model 2 & 21.94 & 21.91 & 1.1 & 98.58 & 92.27 \\
SCDH model 3 & 21.94 & 21.9 & 1.1 & 98.72 & 92.73 \\
SCDH sham 1 & 21.94 & 21.89 & 1.09 & 98.16 & 90.86 \\
SCDH sham 2 & 21.94 & 21.89 & 1.09 & 98.06 & 90.5 \\
SCDH sham 3 & 21.94 & 21.92 & 1.1 & 98.55 & 92.16 \\
\hline
\end{tabular}

performed to determine if re-sequencing was needed. If the alignment result passed QC, downstream analysis including gene expression, differentially expressed genes, cluster analysis, Gene Ontology (GO) enrichment analysis, Kyoto Encyclopedia of Genes and Genomes (KEGG) pathway enrichment analysis, etc. was proceeded with.

\section{Cluster analysis and screening of differentially expressed genes}

Distances of expressed genes were calculated using the Euclidean method [24]. The sum of the squared deviations algorithm was used to calculate distance. The cluster analysis and heat map visualization of gene expression patterns were performed using the "pheatmap" package in the R software of Bioconductor. Differentially expressed mRNAs with statistical significance were identified through Scatter Plot filtering as we reported before [25]. The threshold required for the results to be considered significant was as follows: $q$ value $\leq 0.01$ and absolute value of $\mid \log _{2}$ (Fold Change) $\mid \geq 1.0$ as in our previous study [13].

\section{Functional enrichment analysis of DEGs}

Functional enrichment analysis was performed by functional annotation package "clusterProfiler" in $\mathrm{R}$ studio software (RStudio, Boston, MA). GO and KEGG enrichment analysis was also conducted. For each enriched function term, the $p$ value of enriched functions and the $p$ value by multiple testing corrections were calculated out by "clusterProfiler" package in $\mathrm{R}$ studio software. The GO functional and KEGG pathway enrichment analysis were performed for DEGs using the Data-base for Annotation, Visualization and Integrated Discovery (DAVID) online tools (http://www.geneontology.org and http://www.genome.jp/kegg).

\section{GSEA analysis}

Gene set enrichment analysis (GSEA) was performed according to methods previously described [26]. The gene sets for astrocyte activation (systematic name, M23832), microglia activation (systematic name, M25223), and oligodendrocyte differentiation (systematic name, M15768) were downloaded from the Molecular Signatures Database v7.1 (https://www. gsea-msigdb.org/gsea/msigdb/index.jsp). A FDR $\leq 0.25$ was adopted as the criterion for judging significance [26].

\section{Real-time quantitative PCR (qPCR) analysis}

Total RNA was extracted from the dorsal horn tissue using Trizol reagent (Invitrogen, Carlsbad, USA) according to the manufacturer's protocol. Primer sequences are listed in Table 2. qPCR was performed using the Fast Start Universal SYBR Green Master kit (TaKaRa Bio Inc, China) with $25 \mu$ reaction system according to the manufacturer's protocol by CFX96 Real-Time System (BioRad, USA). Each reaction was performed in triplicates and normalized to Gapdh gene expression. The CT value of each well was determined using the CFX96 Real-Time System software and the average of the triplicates was calculated. The relative quantification was determined by $\Delta \Delta C T$ method $[27,28]$.

\section{Source of microarray data}

Two independent datasets of neuropathic pain models were selected for the study: spared nerve injury (SNI) and chronic constriction injury (CCI) neuropathic microarray. The SNI datasets (GSE18803) were downloaded from Gene Expression Omnibus (GEO) dataset, at the website of https://www.ncbi.nlm.nih.gov/geo/. The CCI datasets comes from a recently published article on CCI sequencing [29]. Differentially expressed genes from these microarray datasets were screened based on criteria as $q$ value $\leq 0.01$ and absolute value of $\mid \log _{2}$ (Fold Change) $\mid \geq 1$.0.

\section{Protein-protein interaction (PPI) network analysis}

The Search Tool for the Retrieval of Interacting Genes (STRING) is used to provide information regarding predicted and experimental interactions of proteins and the prediction method of this database is from neighborhood, gene fusion, co-occurrence, co-expression experiments, databases, and text mining. By setting the combination score $>0.4$ as the reliability threshold value, the web-based STRING database (http://string-db.org/) was used to produce PPI predictions after uploading the union gene list to the search bar [30]. Based on the interplayed relationships, a PPI network was established and then visualized using the Cytoscape software [31]. 
Table 2 The primers used in GPCR

\begin{tabular}{llll}
\hline Primers & Forward & Reverse & Amplicon size (bp) \\
\hline Cxcl13 & CGACTTTGAAAGGTTGCTTGTA & ACACTGGATGAATAGGAAACGT & 219 \\
Nlrp3 & GAGCTGGACCTCAGTGACAATGC & ACCAATGCGAGATCCTGACAACAC & 146 \\
Reg3b & GCTCTCCTGCCTGATGCTCTTATC & AGGTGTCCTTCAGGTCTCTTCTGG & 186 \\
Itgam & GCATCAGTAGCCAGCATCAGTACC & CCGTCCATTGTGAGATCCTTGCC & 119 \\
C3 & TCTGCCTATGCTGCCTTCAACAAC 13 & TGAATCACTGGTCCGTCCTCCTG & 188 \\
LOC100911956 & CAACTGTTCCTGAACTCAACTG & CCTCATCGCCACCGTTGTTCC & 281 \\
Fam111a & AGGCAGCAACAGTATCGTGACTC & ACCAGCTCCTTGCTTGCTCAATG & 197 \\
Mfap4 & ACACCGAATCACAGCGACTTAACC & CTCCGCACTGACCGCATTGG & 143 \\
Gapdh & CACCTCCTGACACTGAAGCAGAAG & AGCCCAGGATGCCCTTTAGT & 120 \\
\hline
\end{tabular}

The connectivity degree of each protein, namely the number of proteins it connected, was calculated to evaluate its importance in this network.

\section{Drug application}

The Specific NLRP3 inflammasome inhibitor MCC950 (APExBIO Technology, TX, USA) was prepared in stock in DMSO and diluted in PBS (1:1000) prior to use. It was applied via intrathecal catheter $(30 \mu \mathrm{g} / \mathrm{rat} /$ day, $12.5 \mu \mathrm{l}$ injection volume). Vehicle containing $(0.1 \%$ DMSO in PBS) was used as control. MCC950 or corresponding vehicle was applied $1 \mathrm{~h}$ before the behavioral test. MCC950 was applied via lumbar catheterization as we described before [32]. Briefly, rats were anesthetized with sodium pentobarbital $(50 \mathrm{mg} / \mathrm{kg}$, i.p.). The lumbar part was shaved and disinfected using $75 \%$ alcohol beforehand. A small incision was cut along L4-5 lumbar vertebrae. The intervertebral foramen was exposed after cutting intervertebral ligament. A PE-10 catheter prefilled with sterilized PBS was inserted into the subarachnoid space. The proper insertion of the catheter was indicated by tail-flick or paw retraction. After the insertion, the proper intraspinal location was examined by lidocaine $(1 \%, 10 \mu \mathrm{l} / \mathrm{rat})$ injection via a catheter. The proper intraspinal location of the catheter was indicated by a quick motor paralysis of the hind limbs of the rat, usually lasting $15-30 \mathrm{~min}$. The catheter was then secured and skin incision was sutured. The rats were placed back into individual cages for further recovery.

\section{Western blot}

Rats were sacrificed after behavioral testing on days 7 . Rats were deeply anesthetized using pentobarbital (50 $\mathrm{mg} / \mathrm{kg}$, i.p.) and transcardially perfused with $200 \mathrm{~mL}$ normal saline $\left(4^{\circ} \mathrm{C}\right)$. The lumbar spinal cord was immediately removed and stored at $-80^{\circ} \mathrm{C}$. Tissues were homogenized in RIPA buffer $(50 \mathrm{mM}$ Tris [pH 7.4], 150 $\mathrm{mM} \mathrm{NaCl}, 1 \%$ Triton X-100, 1\% sodium deoxycholate, sodium orthovanadate, $0.1 \%$ SDS, EDTA, sodium fluoride, leupeptin, and $1 \mathrm{nM}$ PMSF) and then centrifuged at $15,000 \mathrm{rpm}$ for $15 \mathrm{~min}$ at $4{ }^{\circ} \mathrm{C}$ and the supernatant was collected. The protein concentration was determined using the BCA method according to the kit's instruction (Thermo Fisher, USA) and $20 \mu \mathrm{g}$ protein was loaded in each lane. Protein samples were separated on 8-12\% SDS-PAGE gels and electrophoretically transferred to polyvinyl difluoride (PVDF) membranes (Merck KGaA, Darmstadt, Germany). The membranes were blocked with $5 \%$ non-fat milk at room temperature for $1 \mathrm{~h}$, followed by overnight incubation at $4{ }^{\circ} \mathrm{C}$ with the following primary antibodies diluted in blocking buffer: IL-1 $\beta$ (1:500, rabbit polyclonal, \#ab9722, Abcam), NLRP3 (1:500, rabbit polyclonal, \#NBP2-12446, Novus), Caspase-1 (1:500, rabbit polyclonal, ab1872, Abcam). Subsequently, the immunoblots were incubated with the 2 nd antibodies for $1 \mathrm{~h}$ at room temperature. Mouse anti$\beta$-actin (HRP Conjugate, 1:5000, \#ab20272, Abcam) was used as reference control. The immunoreactivity was detected using enhanced chemiluminescence (BIO-RAD, USA) and visualized with an Image Quant LAS 4000 (EG, USA). The density of each band was measured using Image Quant TL 7.0 analysis software (GE, USA). The expression level of the target protein was normalized with $\beta$-actin protein expression level.

\section{Statistical analysis}

Data in graphs are expressed as means \pm SEM. One- or two-way ANOVA followed by Tukey's post hoc test was used for comparison among groups $\geq 3$. Student's $t$ test was used for comparisons between two groups. Comparison is considered significantly different if the $p$ value $<0.05$.

\section{Results}

CPIP model rats showed persistent mechanical allodynia and glial activation in the spinal cord dorsal horn

We first established the rat model of CPIP to mimic human CRPS-I according to methods described before [9]. 
As shown in Fig. 1a-c, the ipsilateral hind paw showed obvious edema compared with the contralateral hind paw $3 \mathrm{~h}$ after ischemia and returned normal 3 days later. In contrast, CPIP model rats displayed long-lasting (> 14 days) mechanical allodynia in both ipsilateral and contralateral hind paws (Fig. 1d, e). These signs are consistent with previous publications and suggested successful establishment of the CPIP model [9]. We sacrificed the rats and collected the spinal cord dorsal horn at day 7 and examined the expression pattern of GFAP and OX-42, markers for astrocyte and microglia, respectively. As shown in Fig. 2 a-d, the expression of GFAP and OX-42 were both significantly increased in the ipsilateral $\mathrm{SCDH}$ of CPIP model rats compared with sham rats, an indication of glial cell activation in SCDH of CPIP model.

\section{Examining gene expression profiles of SCDH after CPIP by RNA-Seq}

In order to explore the genes that possibly participate in pain mechanisms of CPIP model, we harvested L4-6 SCDH of CPIP model and sham rats and analyzed the gene expression profiles via RNA-Seq. The RNA-Seq generated 21.94 million raw reads per sample and the clean reads Q20\% reached nearly 99.0\% (Table 1). More than $90 \%$ of bases reached a quality score $\geq$ Q30 and over $95 \%$ of the clean reads data were mapped to the rat genome (Table 1). Eventually, 18,672 genes were

\section{A}

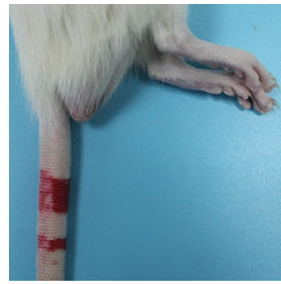

Before ischemia

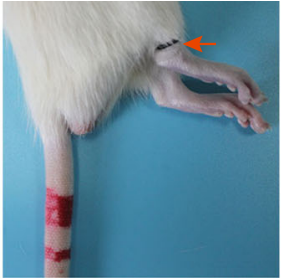

Ischemia

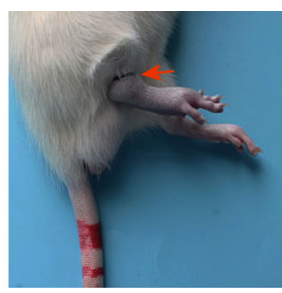

Post 3 hours

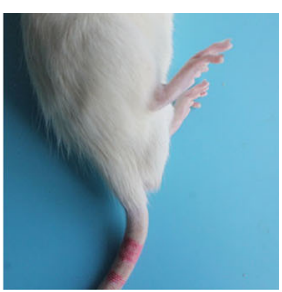

Post 7 days
B

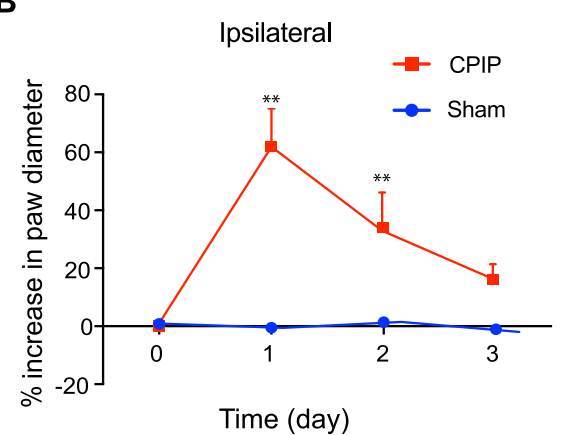

D

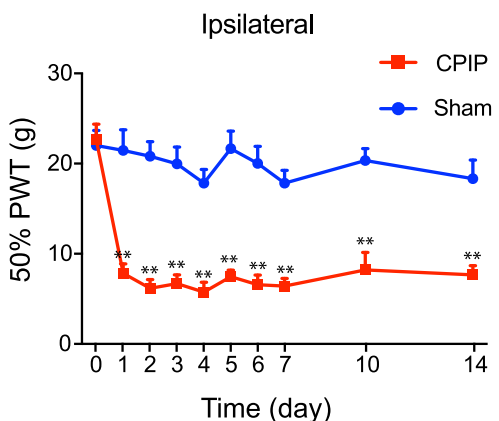

C

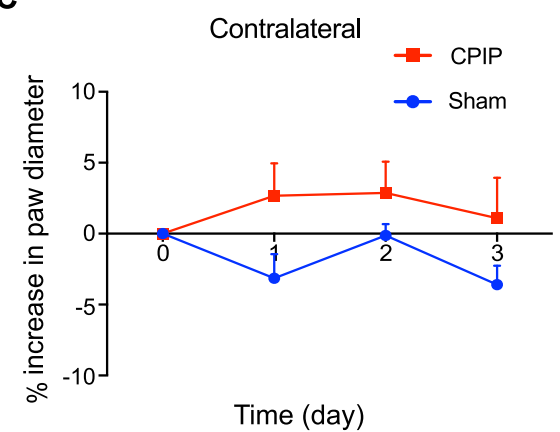

$\mathbf{E}$

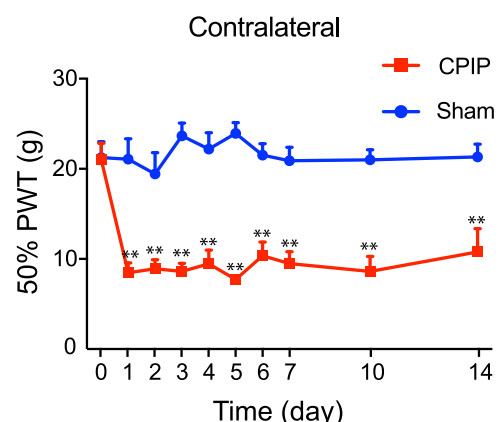

Fig. 1 The chronic post-ischemia pain (CPIP) rat model exhibits persistent mechanical allodynia. a Representative photographs of rat hind paw taken before the CPIP model establishment, during ischemia, $3 \mathrm{~h}$ and 7 days after the model establishment. The red arrow indicates the O-ring clamped right above the ankle. b, c The paw swelling of ipsilateral and contralateral hind paws of CPIP and Sham group rats. d, e 50\% paw withdraw threshold (PWT) of ipsilateral and contralateral hind paws of sham and CPIP group rats. ${ }^{* *} p<0.01$ vs. sham group. $n=8$ rats/group. Two-way ANOVA followed by Tukey's post hoc test was used for comparison 
A
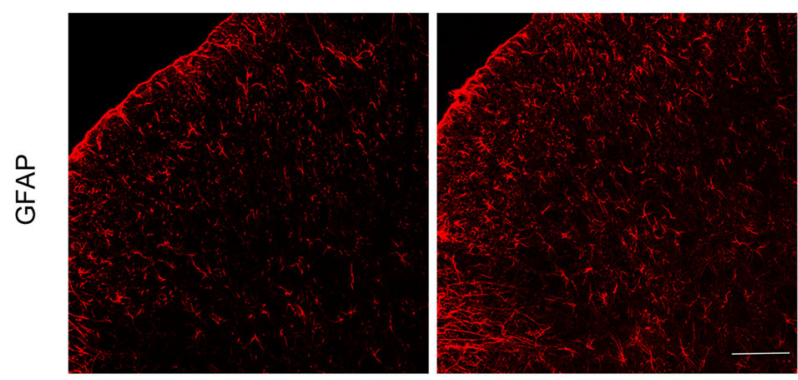

Sham

CPIP

B

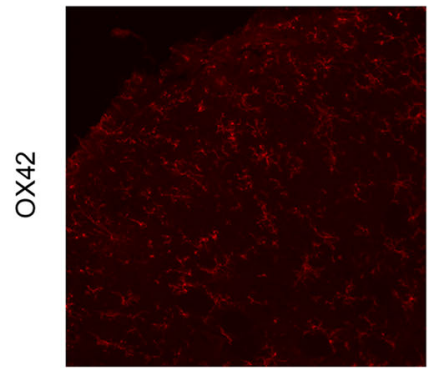

Sham

C

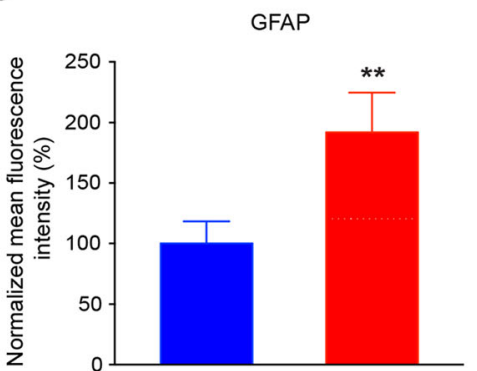

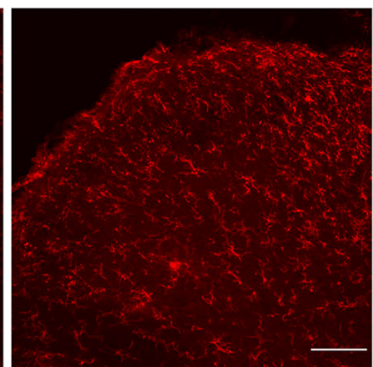

CPIP
D

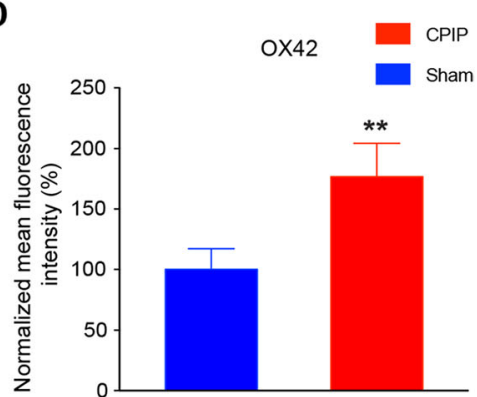

Fig. 2 Astrocytes and microglia are activated in ipsilateral SCDH of CPIP model rats. a The expression of GFAP in ipsilateral SCDH of sham and CPIP group. $\mathbf{b}$ The expression of OX-42 in ipsilateral SCDH of sham and CPIP group. $\mathbf{c}$, $\mathbf{d}$ The summarized normalized \% increase in the mean fluorescence intensity of GFAP (c) and OX-42 staining (d). The value was normalized to sham group. Scale bar indicates $50 \mu \mathrm{m} .{ }^{* *} p<0.01$ vs. sham group. Student's $t$ test was used for comparisons

successfully mapped and identified from RNA-Seq (Additional file 1: Suppl. Table 1). We then set to identify differentially expressed genes (DEGs) with criteria of fold change $\geq 2$ and $q$ value $\leq 0.01$. Based upon this criterion, we identified a total of 435 DEGs (including 324 up- and 111 downregulated), which are further displayed in the scatter plot graph (Fig. 3a, Additional file 2: Suppl. Table 2). The identified DEGs were then summarized and plotted in the heat map (Fig. 3b). Cluster analysis indicated a high level of concordance within both CPIP and Sham group samples and a clear segregation between the two groups (Fig. 3b).

\section{Analysis of DEGs in SCDH of CPIP model rats}

Among the DEGs that we identified, some of the genes are known to be related with inflammation or pain processes, such as Itgam (integrin subunit alpha $\mathrm{M}$, fold change $=261.4)$, Cxcl13 $(\mathrm{C}-\mathrm{X}-\mathrm{C}$ motif chemokine 13 precursor, fold change $=54.9)$, C3 (complement component 3 , fold change $=7.1), C d 68$ (Cd68 molecule, fold change $=5.2$ ), and $I l-1 \beta$, (interleukin-1 beta, fold change $=3.8$ ). We further analyzed the data and found that 77 genes showed expression changes more than 10-fold, with 31 upregulated genes and 46 downregulated gene, such as Isoc1 (isochorismatase domain-containing protein 1 , fold change $=845.7$ ), Stra 8 (stimulated by retinoic acid 8, fold change $=192.4$ ), Rpl30 (ribosomal protein L30, fold change $=115.7$ ); 33 genes showed expression changes between 5 - and 10-fold, which include 24 upregulated and 9 downregulated genes. The detailed information of the top 20 up- and 20 downregulated DEGs are summarized in Tables 3 and 4, respectively. 

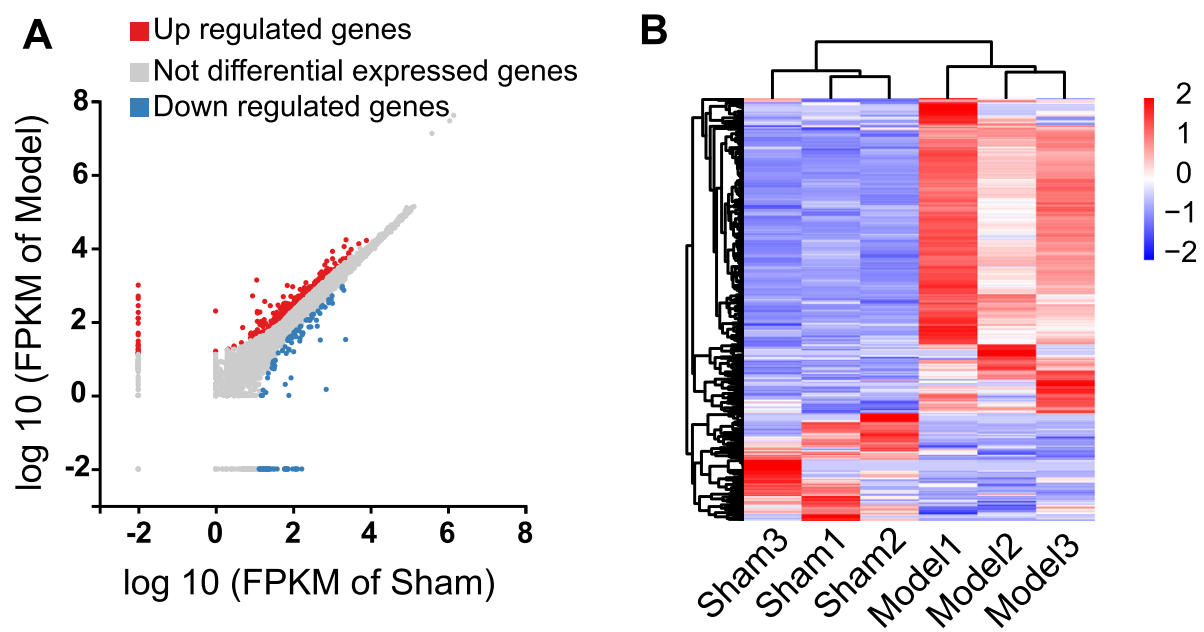

Fig. 3 RNA-Seq reveals gene expression changes in SCDH induced by CPIP. a Scatter plot showing gene expression profiles in ipsilateral SCDH of CPIP group compared with sham group. Red and blue spots indicate up- and downregulated DEGs, respectively, whereas gray spots indicate non-DEGs. b Heat map displaying the hierarchical clustering of DEGs from CPIP and sham group

Table 3 The detailed information of the top 20 upregulated DEGs

\begin{tabular}{|c|c|c|c|c|c|}
\hline $\begin{array}{l}\text { Upregulated } \\
\text { gene }\end{array}$ & Gene ID & Location & $\begin{array}{l}\text { Log2 fold change (CPIP/ } \\
\text { Sham) }\end{array}$ & $Q$ value & Official gene name (NCBI) \\
\hline LOC100363502 & 100363502 & $62475574-62476771$ & 10.9252125 & $9.77 E-139$ & \\
\hline LOC103689943 & 103689943 & $28267-72912$ & 9.9331474 & 2.03E- 81 & \\
\hline Isoc1 & 364879 & $53727203-53747612$ & 9.72392644 & $1.35 \mathrm{E}-72$ & Isochorismatase domain-containing protein 1 \\
\hline LOC100911615 & 100911615 & $\begin{array}{l}213997721- \\
214002811\end{array}$ & 9.07094965 & $1.79 \mathrm{E}-50$ & \\
\hline LOC100911825 & 100911825 & $91219838-91237274$ & 8.61974834 & $2.04 \mathrm{E}-24$ & \\
\hline LOC685963 & 685963 & $\begin{array}{l}164002409- \\
164002748\end{array}$ & 8.46420752 & $9.52 \mathrm{E}-36$ & \\
\hline LOC103689965 & 103689965 & $4495440-4508232$ & 7.92974175 & $3.41 \mathrm{E}-26$ & \\
\hline Stra8 & 500079 & $62447724-62474802$ & 7.58808827 & $2.55 \mathrm{E}-42$ & Stimulated by retinoic acid 8 \\
\hline LOC100909795 & 100909795 & $78550661-78577178$ & 7.48236683 & $4.25 \mathrm{E}-20$ & \\
\hline Rpl30 & 64640 & $73213538-73216431$ & 6.85524869 & 0 & Ribosomal protein L30 \\
\hline LOC108348184 & 108348184 & $1622570-1683919$ & 6.59189138 & $9.95 \mathrm{E}-12$ & \\
\hline LOC103690173 & 103690173 & $3738-18580$ & 6.44436116 & $1.03 \mathrm{E}-10$ & \\
\hline LOC103694874 & 103694874 & $23060-31871$ & 5.87292704 & $1.62 \mathrm{E}-07$ & \\
\hline Cxcl13 & 498335 & $15253146-15258221$ & 5.78195338 & $1.23 \mathrm{E}-117$ & $\mathrm{C}-\mathrm{X}-\mathrm{C}$ motif chemokine ligand 13 \\
\hline Rsph4a & 309767 & $32450701-32467362$ & 5.59130694 & $2.57 \mathrm{E}-06$ & Radial spoke head component 4A \\
\hline LOC100912393 & 100912393 & 3940948-3965964 & 5.45532247 & $8.21 \mathrm{E}-06$ & \\
\hline $\operatorname{Rps} 20$ & 122772 & $16706052-16707214$ & 5.41559782 & $1.14 \mathrm{E}-05$ & Ribosomal protein \$20 \\
\hline Batf & 299206 & $\begin{array}{l}109562415- \\
109584541\end{array}$ & 5.13596145 & 8.93E- 05 & $\begin{array}{l}\text { Basic leucine zipper ATF-like transcription } \\
\text { factor }\end{array}$ \\
\hline Aqp3 & 65133 & $57423735-57429252$ & 5.13596145 & $8.93 \mathrm{E}-05$ & Aquaporin-3 \\
\hline Crkl & 287942 & $87338606-87356644$ & 4.94331637 & 0.00030049 & Crk-like proto-oncogene, adaptor protein \\
\hline
\end{tabular}




\section{RNA-Seq data validation using $\mathrm{qPCR}$}

Next, we set to examine the reliability of our RNA-Seq data using qPCR. We randomly selected three upregulated genes (LOC100911956, Reg3b, and Irf8) and two downregulated genes (Fam111a and Mfap4) from the DEGs list and verified their expression via qPCR. The results of $\mathrm{qPCR}$ showed that the expression of LOC100911956, Reg3b, and Irf8 were all significantly upregulated, whereas Fam111a and Mfap4 were both significantly downregulated in ipsilateral SCDH of CPIP model rats $v s$. sham rats (Fig. $4 \mathrm{a}, \mathrm{b}$ ), which are consistent with RNA-Seq data. Furthermore, we continued to examine some representative genes which are well documented to participate in neuroinflammation or pain mechanisms, including Il1 $\beta$, Itgam, and C3, by qPCR. The qPCR data suggested that the expression of $I l 1 \beta$, Itgam, and C3 were all significantly upregulated in SCDH of CPIP model rats vs. sham rats (Fig. 4c), consistent with the RNA-Seq data. Thus, the results of qPCR provide evidence showing that the RNA-Seq data for gene expression profiling was reliable.

\section{Function and pathway analysis of DEGs in SCDH of CPIP model rats}

In order to further explore the mechanisms underlying CRPS-I, we carried out gene ontology (GO) analysis of the DEGs in SCDH of CPIP and sham group of rats. According to functional annotation in GO database, we found that the most significantly enriched biological process of upregulated DEGs in CPIP model group were inflammatory response, innate immune response, defense response to virus, etc. (Fig. 5a, Additional file 3: Suppl. Table 3). The most significantly enriched cellular component of upregulated DEGs in CPIP model group was immunological synapse, external side of plasma membrane and NADPH oxidase complex, etc. (Fig. 5b, Additional file 4: Suppl. Table 4). The most significantly enriched molecular function of upregulated DEGs in CPIP model group was superoxide-generating NADPH oxidase activity, IgG binding, endopeptidase inhibitor activity, etc. (Fig. 5c, Additional file 5: Suppl. Table 5). Meanwhile, the most significantly enriched biological process of downregulated DEGs in CPIP model group was oxygen transport, response to organic substance, translation, etc. (Fig. 5d, Additional file 6: Suppl. Table 6). The most significantly enriched cellular component of downregulated DEGs in CPIP model group was hemoglobin complex, cytosolic small ribosomal subunit and haptoglobin-hemoglobin complex, etc. (Fig. 5e, Additional file 7: Suppl. Table 7). The most significantly enriched molecular function of downregulated DEGs in CPIP model group was oxygen transporter activity,

Table 4 The detailed information of the top 20 downregulated DEGs

\begin{tabular}{|c|c|c|c|c|c|}
\hline $\begin{array}{l}\text { Downregulated } \\
\text { gene }\end{array}$ & Gene ID & Location & $\begin{array}{l}\text { Log2 fold change } \\
\text { (CPIP/Sham) }\end{array}$ & $Q$ value & Official gene name (NCBI) \\
\hline Rps6 & 29304 & $105197821-105200681$ & -8.9793272 & $3.56 \mathrm{E}-136$ & Ribosomal protein $\mathrm{S} 6$ \\
\hline LOC102549712 & 102549712 & $48508746-48542941$ & -8.4277401 & $3.35 \mathrm{E}-34$ & \\
\hline LOC100910581 & 100910581 & 21987993-21989200 & -8.0682673 & $9.43 \mathrm{E}-28$ & \\
\hline LOC100910446 & 100910446 & $23542376-23560911$ & -7.9258687 & $1.52 \mathrm{E}-25$ & \\
\hline Rp/9 & 29257 & $44524419-44527613$ & -7.9088834 & $2.73 E-25$ & Ribosomal protein L9 \\
\hline Triap 1 & 108348066 & 13511349-13513661 & -7.8860873 & $5.88 \mathrm{E}-25$ & TP53-regulated inhibitor of apoptosis 1 \\
\hline Pddcl & 309110 & $214389006-214394451$ & -7.6510269 & $1.02 \mathrm{E}-21$ & $\begin{array}{l}\text { Glutamine amidotransferase-like class } 1 \\
\text { domain containing } 1\end{array}$ \\
\hline Slc40a1 & 108348131 & $52894300-52913267$ & -7.2422467 & $5.11 \mathrm{E}-17$ & Solute carrier family 40 member 1 \\
\hline LOC688583 & 688583 & $51458436-51462755$ & -7.2391214 & $5.50 E-17$ & \\
\hline LOC102550456 & 102550456 & 21748608-21761500 & -7.1733103 & $2.53 E-16$ & \\
\hline Conj & 294053 & 259926537-259944275 & -7.0687075 & $2.52 \mathrm{E}-15$ & Cyclin J \\
\hline Snip1 & 108348099 & 143063206-143070899 & -7.0547769 & $3.38 \mathrm{E}-15$ & Smad nuclear interacting protein 1 \\
\hline LOC108348298 & 108348298 & 4487809-4499727 & -6.9606747 & $2.37 \mathrm{E}-14$ & \\
\hline LOC108349606 & 108349606 & $166282044-166282845$ & -6.9454155 & $3.21 \mathrm{E}-14$ & \\
\hline Scgbla1 & 25575 & $225279698-225283246$ & -6.3193658 & $9.30 \mathrm{E}-19$ & Secretoglobin family $1 \mathrm{~A}$ member \\
\hline$R p / 35 a$ & 57809 & $7186459-7186874$ & -6.3178853 & $1.09 \mathrm{E}-09$ & Ribosomal protein L35a \\
\hline LOC100360679 & 100360679 & $104521700-104522246$ & -6.1465009 & 0 & \\
\hline LOC103689971 & 103689971 & 35396229-35518693 & -6.0905471 & $2.02 \mathrm{E}-08$ & \\
\hline Prss 1 & 24691 & 70776046-70779249 & -5.9881599 & $6.66 \mathrm{E}-08$ & Serine protease 1 \\
\hline Sftpal & 24773 & 18716019-18719404 & -5.7888511 & $5.52 \mathrm{E}-07$ & Surfactant protein A1 \\
\hline
\end{tabular}



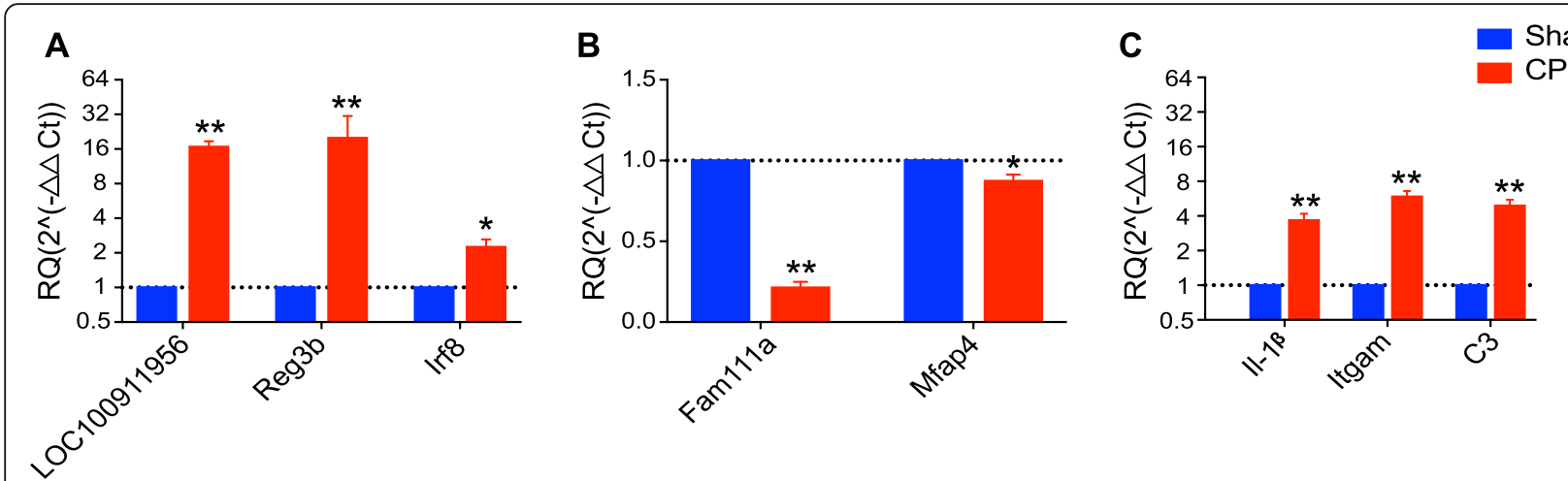

Fig. 4 The validation of RNA-Seq results via qPCR. a The expression of three randomly selected upregulated DEGs from RNA-Seq was examined by GPCR. $\mathbf{b}$ The expression of two randomly selected downregulated DEGs from RNA-Seq was examined by qPCR. $\mathbf{c}$ The expression of some typical genes involved in inflammation and pain was examined by $q P C R . n=6$ rats/group. ${ }^{*} p<0.05,{ }^{* *} p<0.01$ vs. sham group. Student's $t$ test was used for comparisons

oxygen binding and heme binding, etc. (Fig. 5f, Additional file 8: Suppl. Table 8).

We further performed KEGG pathway enrichment analysis of the DEGs between CPIP model and sham groups. Scatter diagrams of KEGG measured the degree of enrichment by rich factor, $q$ value and gene number of the enriched pathway. Based upon the KEGG pathway enrichment analysis, we found that the DEGs in SCDH of CPIP model group were mainly related with phagosome, osteoclast differentiation, tuberculosis, complement and coagulation cascades and phagocytosis, etc. (Fig. 6, Additional file 9: Suppl. Table 9).

\section{Comparison of RNA-Seq dataset of CPIP model with other neuropathic pain model datasets}

In our previous study, we compared DEGs of DRGs from CPIP model rats with other well-established neuropathic or inflammatory pain models and found that CPIP model exhibited more changes in neuropathicpain-related genes than inflammatory-pain-related genes [13]. In the present study, we continued to compare our RNA-Seq data derived from SCDH of CPIP model rats with the datasets obtained from two other wellestablished rat models of neuropathic pain, namely, the SNI and CCI models. Two gene profiling datasets of CCI and SNI models were downloaded as described in the "Materials and methods" section. We imposed the same criteria (fold change $\geq 2$ or $\leq-2, q \leq 0.01$ ) upon both SNI and CCI datasets for identifying DEGs. We found that the DEGs of CPIP model group had 46 and 40 genes overlapping with the DEGs of SNI and CCI model, respectively, accounting for $10.6 \%$ and $9.2 \%$ of all DEGs of CPIP model (Fig. 7a, Additional file 10: Suppl. Table 10, Additional file 11: Suppl. Table 11). Besides, the three groups shared a core set of 24 genes that were overlapped together (Fig. 7a, Table 5). These 24 genes all showed upregulation in the SCDH of CPIP model group. Finally, in order to study the interactions and the hub genes of DEGs that are involved in CPIP and other neuropathic pain models, we carried out PPI network analysis of these 24 genes and found that the major hub genes deduced from PPI analysis include Cd53, Aif1, C1qc, C1qa, C1qb, Fcgr3a, and Fcgr2b (Fig. 7b).

In order to explore which cell type in SCDH is particularly affected by CPIP model, we performed gene set enrichment analysis (GSEA) using gene sets wellestablished for astrocyte activation (systematic name, M23832), microglia activation (M25223), and oligodendrocyte differentiation (M15768) from the Molecular Signatures Database v7.1. We found that the CPIP model showed significant activation of the three gene sets by GSEA analysis (NES > 1.0, FDR $\leq 0.25$ ), indicating that all three cell types in the SCDH are affected by CPIP model (Suppl. Fig. 1A-C). As a comparison, we also performed GSEA analysis of the SNI neuropathic pain model, in which the activation of astrocytes and microglia in SCDH are well established. As a result, GSEA analysis revealed that SNI model showed similar activation of the three gene sets compared with CPIP model (Suppl. Fig. 1D-F). Therefore, GSEA analysis suggests that the microglia, astrocytes, and oligodendrocytes in SCDH are all significantly affected in CPIP model.

\section{Identifying NLRP3 inflammasome in SCDH as a key player in mediating the pain and inflammation responses in CPIP rat model.}

We observed $I l-1 \beta$ gene is significantly upregulated in SCDH of CPIP rats (Fig. 4c). IL-1 $\beta$ overproduction in $\mathrm{SCDH}$ has recently been shown to be important for the neuroinflammation and pain response of a mouse model of CRPS-I [33]. However, the precise mechanisms of how IL- $1 \beta$ is overproduced under CRPS-I is still unknown. NLRP3 inflammasome is a complex of proteins responsible for the cleavage of pro-IL-1 $\beta$ into active IL- 
A

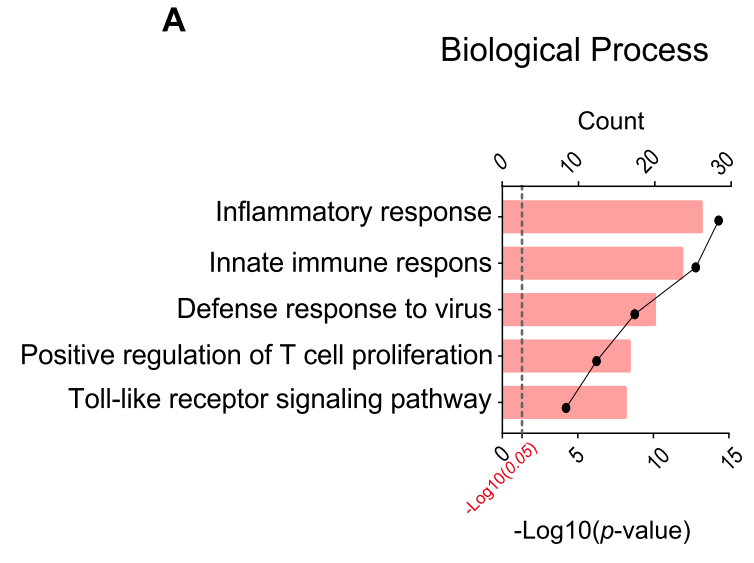

B

Cellular Component

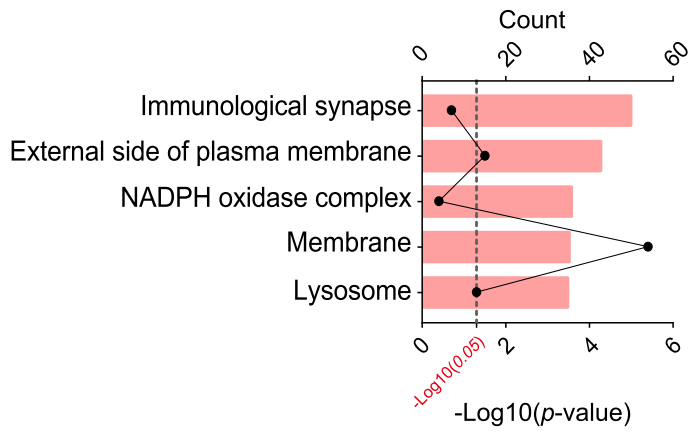

C

Molecular Function

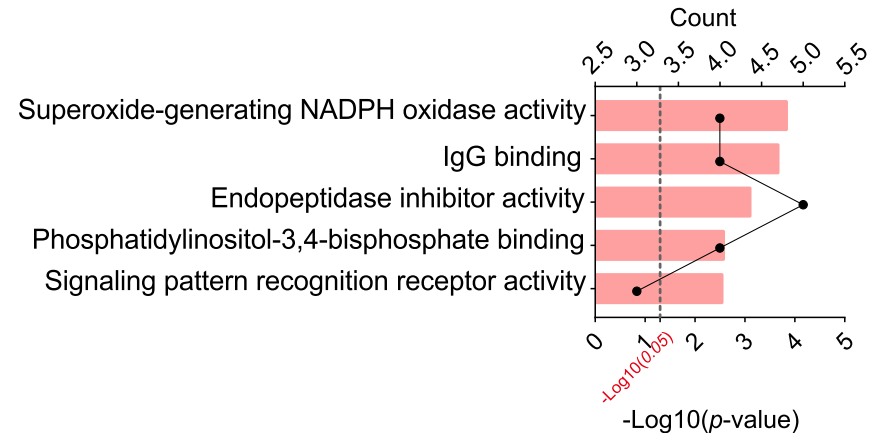

D

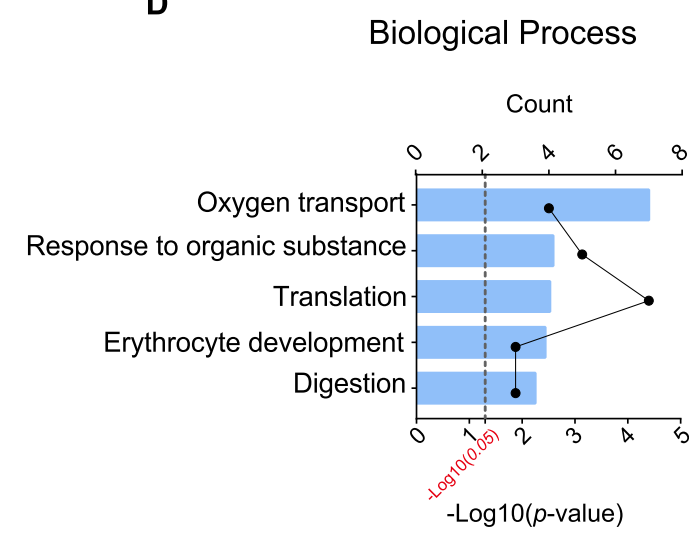

E

Cellular Component

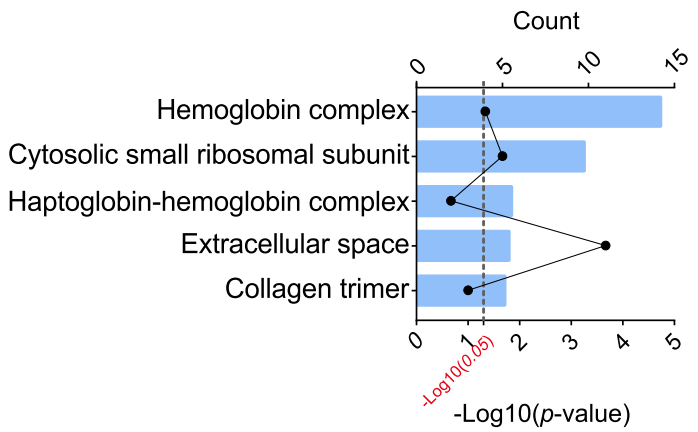

F

Molecular Function

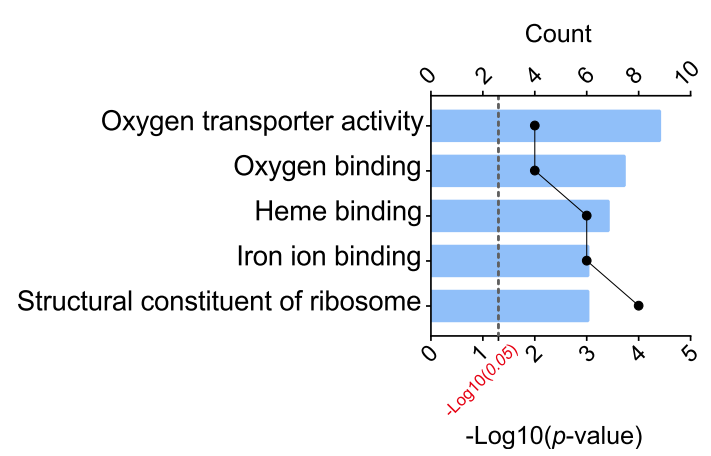

Fig. 5 GO pathway analysis of DEGs. a-c The top 5 significant biological processes, cellular components, and molecular functions of upregulated DEGs. $\mathbf{d}$-f The top 5 significant biological processes, cellular components, and molecular functions of downregulated DEGs. The dotted line indicates the $p$ value of 0.05

$1 \beta$, which plays an important role in mediating pain response, inflammation, and central sensitization [34-37]. We therefore analyzed all NLR family gene changes in SCDH of CPIP model rats vs. sham rats and found that Nlrp3 gene is significantly upregulated (Fig. 8a). In contrast, Nlrp3 gene expression is not significantly altered in CCI or SNI model rats by analyzing the dataset as mentioned in Fig. 7, suggesting Nlrp3 gene expression upregulation in SCDH is specific to CPIP model rats.
We therefore continued to validate the Nlrp3 gene expression by qPCR. qPCR showed that the mRNA expression of the three major components in NLRP3 inflammasome, namely Nlpr3, Caspase-1, and Il-1ß, are all significantly upregulated in ipsilateral SCDH of CPIP model rats 7 days after model establishment (Fig. 8b-d). Moreover, Western blot studies further confirmed that the protein expressions of NLRP3, Caspase- 1 , and IL-1 $\beta$ are all significantly upregulated in ipsilateral SCDH of 


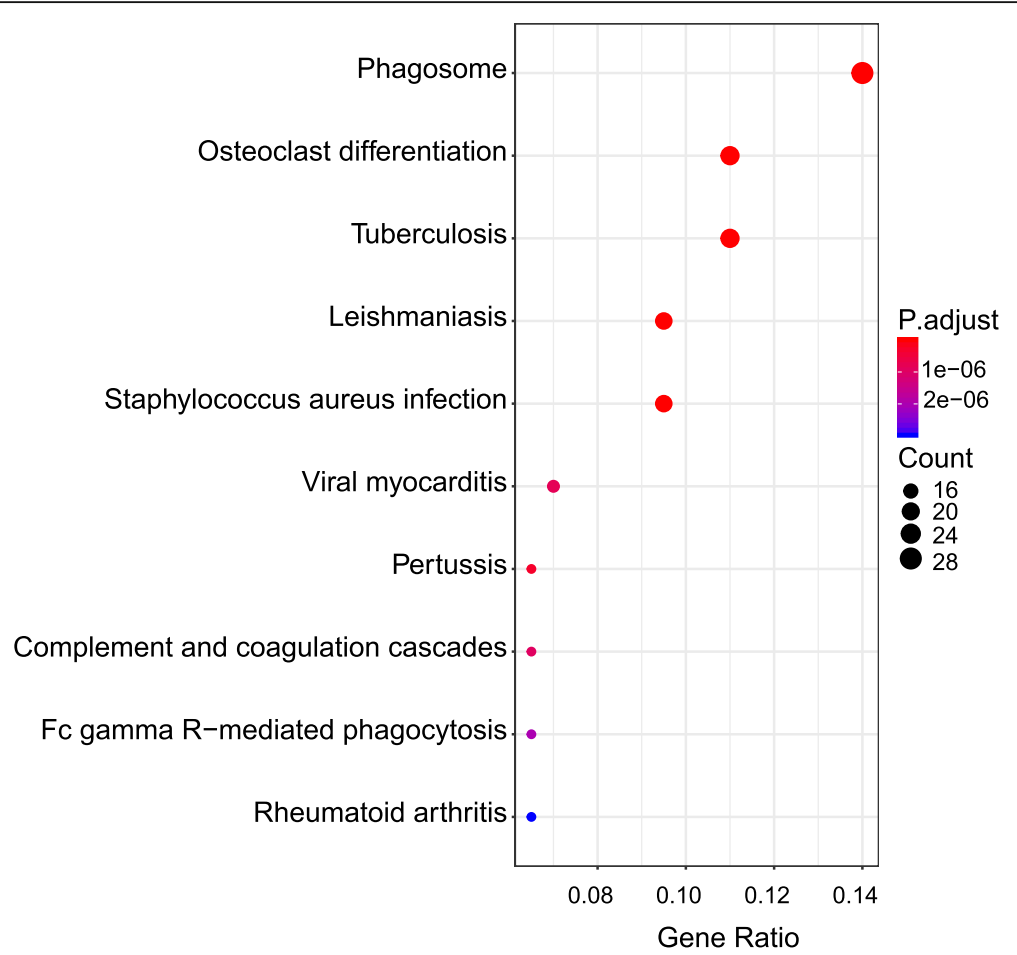

Fig. 6 KEGG pathway analysis of DEGs. Bubble plots showing the significant pathways for up- and downregulated DEGs. Larger bubbles indicate higher number of genes. The color of each bubble reflects significance ( $p$ value)

CPIP model rats 7 days after model establishment (Fig. $8 \mathrm{e}-\mathrm{g})$, suggesting the activation of NLRP3 inflammasome in SCDH of CPIP model rats.

We next examined whether NLRP3 inflammasome contributed to the pain response of CPIP model rats. In order to address this issue, we used MCC950, a potent and specific inhibitor of NLRP3 inflammasome [38, 39]. MCC950 or corresponding vehicle (0.1\% DMSO in PBS) was applied through intrathecal catheter on a daily basis after model establishment (Fig. 9a). As expected, intrathecal MCC950 application significantly reduced the upregulation of NLRP3 and IL-1 $\beta$ overexpression in ipsilateral SCDH of CPIP model rats (Fig. 9b, c). More importantly, intrathecal MCC950 significantly attenuated the mechanical allodynia of ipsilateral hind limbs of CPIP model rats vs. CPIP model rats injected with vehicle only (Fig. 9d). Area under the curve (AUC) analysis further indicated an overall attenuation of the mechanical allodynia by accumulated MCC950 treatment (Fig. 9e). To further study the cellular mechanisms underlying NLRP3 inflammasome in mediating the pain response of CPIP model rats, we investigated whether NLRP3 inflammasome participates in the activation of spinal astrocytes and microglia of CPIP model rats, which are crucial steps involved in central pain transduction and sensitization [40, 41]. Immunostaining found that the overexpression of GFAP or OX42 in ipsilateral SCDH of
CPIP model rats was significantly reduced by intrathecal MCC950 treatment (Fig. 10a-f). Thus, the above data indicated that NLRP3 inflammasome contributes to the mechanical allodynia of CPIP model rats via mechanisms possibly involving activation of astrocytes and microglial in SCDH.

\section{Discussion}

In the present study, we examined the gene expression profiles in ipsilateral L4-6 SCDH of the CPIP model and sham rats through RNA-Seq. We identified a number of DEGs and validated their expressions via qPCR. We further looked into the molecular and cellular functions of these DEGs by applying GO and KEGG analysis and found that the most significantly enriched biological processes of upregulated genes are involved in inflammatory response and innate immune response. We further compared our dataset with other published datasets of common neuropathic pain models and identified a core set of genes and pathways that extensively participate in CPIP and other neuropathic pain models. To our knowledge, this is by far the first study to profile the gene expression changes and key pathways in the SCDH of a rat model of CRPS-I using RNA-Seq.

Previous studies have demonstrated that central sensitization plays a critical role in maintaining chronic pain [42]. There is increasing evidence that central 


\section{A}

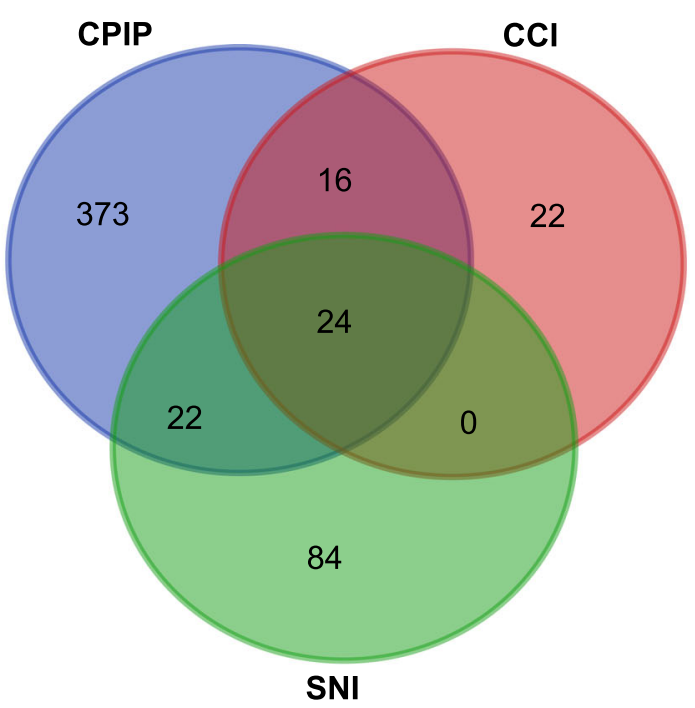

B

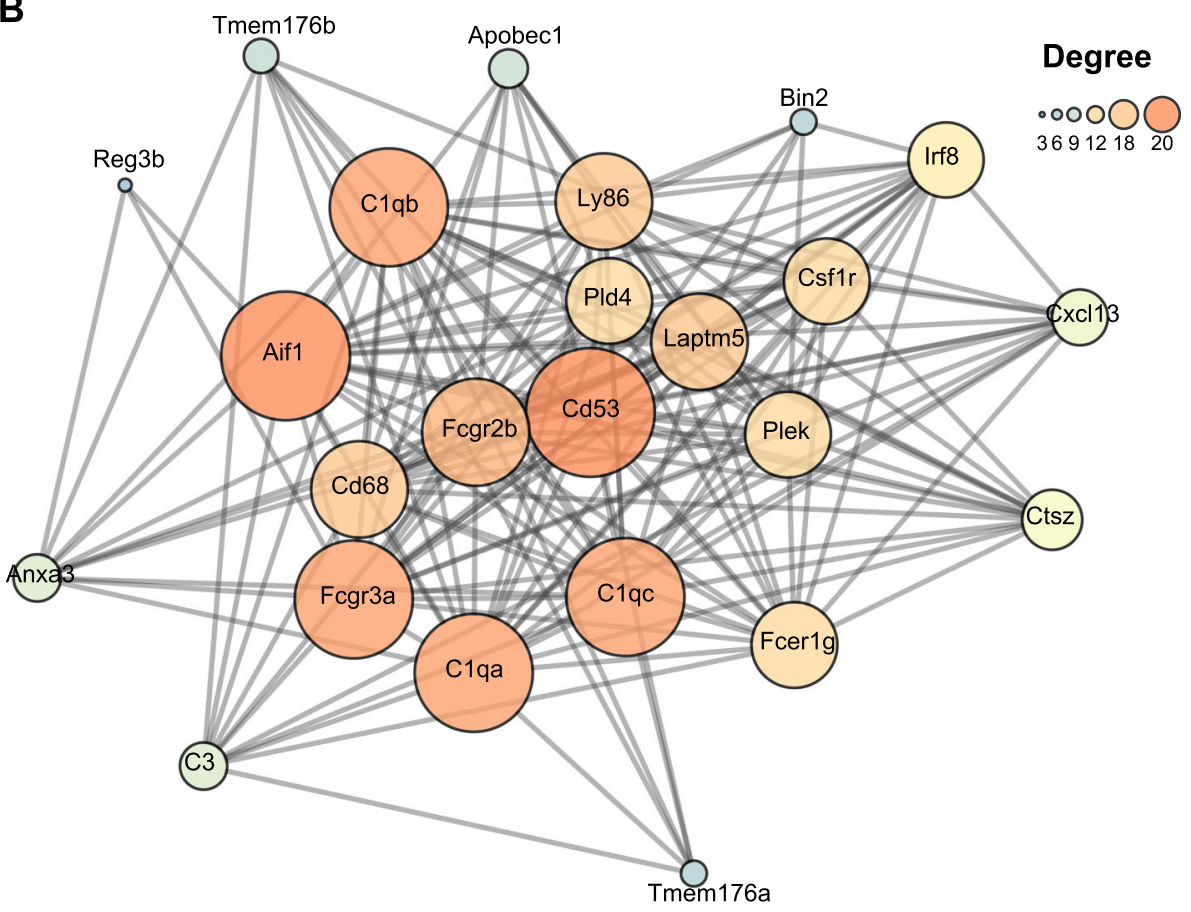

Fig. 7 Comparison of our present CPIP RNA-Seq datasets with other published microarray/RNA-Seq datasets of neuropathic models. a Venn diagram showing the overlapping of DEGs of SCDH from CPIP rat model with rat SNI and CCI neuropathic pain models. $\mathbf{b}$ PPI network analysis of the DEGs overlapped with SNI and CCl gene datasets. Larger circles and darker colors indicate higher degree of interactions

sensitization is driven by neuroinflammation of the surrounding and central nervous system [43]. A key feature of neuroinflammation is the activation of glial cells, such as microglia and astrocytes in the spinal cord and brain, which results in the release of an array of proinflammatory cytokines and chemokines that could sensitize pain-processing neurons in the spinal dorsal horn $[44,45]$. In this study, we found that CPIP model rats showed significant activation of microglia and astrocytes in the ipsilateral SCDH, which is consistent with previous studies and our recent findings [11, 14]. The persistent microglia and astrocytes activation in the $\mathrm{SCDH}$ leads to sustained release of pro-inflammatory cytokines and chemokines in the spinal cord, which contributes to chronic pain condition manifested in CPIP model rats [11]. Therefore, in the present study, we 
Table 5 The 24 DEGs of CPIP overlapping with the SNI and CCI

\begin{tabular}{|c|c|c|}
\hline $\begin{array}{l}\text { Genes } \\
\text { symbol }\end{array}$ & Official gene name(NCBI) & $\begin{array}{l}\text { Change in three } \\
\text { datasets }\end{array}$ \\
\hline Cxcl13 & C-X-C motif chemokine ligand 13 & Up \\
\hline C3 & Complement component 3 & Up \\
\hline Reg3b & $\begin{array}{l}\text { Regenerating family member } 3 \\
\text { beta }\end{array}$ & Up \\
\hline Clqc & Complement $\mathrm{Clq} \mathrm{C}$ chain & Up \\
\hline Cd68 & Cd68 molecule & Up \\
\hline Clqa & Complement C1q A chain & Up \\
\hline Clqb & Complement $\mathrm{C} 1 \mathrm{q}$ B chain & Up \\
\hline Fcgr3a & Fc fragment of IgG receptor Illa & Up \\
\hline Apobec1 & $\begin{array}{l}\text { Apolipoprotein B mRNA editing } \\
\text { enzyme catalytic subunit } 1\end{array}$ & Up \\
\hline Irf8 & Interferon regulatory factor 8 & Up \\
\hline Fcgr2b & Fc fragment of IgG receptor lllb & Up \\
\hline Pld 4 & $\begin{array}{l}\text { Phospholipase D family, member } \\
4\end{array}$ & Up \\
\hline $\operatorname{Bin} 2$ & Bridging integrator 2 & Up \\
\hline Ly86 & Lymphocyte antigen 86 & Up \\
\hline Ctsz & Cathepsin Z & Up \\
\hline Fcer1g & Fc fragment of IgE receptor lg & Up \\
\hline Anxa3 & Annexin A3 & Up \\
\hline Tmem176a & Transmembrane protein 176A & Up \\
\hline Csfir & $\begin{array}{l}\text { Colony stimulating factor } 1 \\
\text { receptor }\end{array}$ & Up \\
\hline Aif1 & Allograft inflammatory factor 1 & Up \\
\hline Tmem176b & Transmembrane protein 176B & Up \\
\hline Plek & Pleckstrin & Up \\
\hline Cd53 & Cd53 molecule & Up \\
\hline Laptm5 & $\begin{array}{l}\text { Lysosomal protein } \\
\text { transmembrane } 5\end{array}$ & Up \\
\hline
\end{tabular}

performed the expression profiling of the SCDH of CPIP model rats, aiming to unravel the molecular mechanisms underlying CRPS-I.

GO analyses revealed that the most significantly enriched biological processes of upregulated genes in SCDH of CPIP vs. sham group were related with inflammatory and innate immune response, defense response to virus, positive regulation of $\mathrm{T}$ cell proliferation and Toll-like receptor signaling pathway, etc. This result implies that neuroinflammation in SCDH may constitute a predominant mechanism involved in the pathophysiology of the CRPS-I. Besides, GO analysis of molecular function revealed that superoxide-generating NADPH oxidase activity is among the most significantly enriched function in the SCDH of CPIP model rats. NADPH oxidases are the main enzymes that produce reactive oxygen species (ROS) and trigger subsequent oxidative stress. NADPH oxidase-mediated ROS production and oxidative stress are involved in persistent pain, including neuropathic and inflammatory pain [46, 47]. Reducing ROS generation alleviates pain in CPIP model rats [48, 49]. Therefore, the above results suggest that targeting neuroinflammation and NADPH oxidase-mediated ROS production may be potential effective strategies to ameliorate CRPS-I.

The KEGG analysis unraveled that the most enriched pathway the DEGs are involved in is related with phagosome. This result is consistent with our recent findings that phagosome is a predominant process that took place in the peripheral DRGs of CPIP model rats [13]. Similar result was also observed in another study performing RNA-Seq on SCDH of CCI-induced neuropathic pain model [29]. One of the representative genes involved in phagosome is Itgam, a marker for macrophage and microglia. In our study, Itgam gene upregulation is detected by both RNA-Seq and qPCR. We also found that microglia is significantly accumulated in the ipsilateral SCDH of CPIP model rat, a result consistent with other studies $[11,15]$. Accumulated microglia cells release BDNF and increase neuronal excitability in SCDH by via neuron-glial crosstalk, which contributes to pain in CPIP [11]. Therefore, our data, together with the others, suggest that microglia in the SCDH may play an important role in mediating pain symptom and neuroinflammation of CRPS-I.

In the present study, cluster analysis indicated a high level of concordance of datasets within both CPIP and sham group samples and a clear segregation between the two groups. However, we noticed that some gene expression changes in model 1 are more robust than model 2 and 3, which possibly means that some of the gene changes may be driven by a single sample in the CPIP model. Since only 3 biological samples are included in each group, therefore, we have to acknowledge that our present RNA-Seq dataset may possess certain weakness due to the limited number of samples included. However, it should be noted that RNA-Seq is a preliminary data screening method. qPCR and protein assay should be followed for further validation of targets. In our study, we carried out further qPCR and Western blot experiments using different batches of animals to validate our RNA-Seq data and we successfully identified many genes and targets that showed a consistent trend with our RNA-Seq dataset. Therefore, we hope future studies which incorporate more samples into RNA-Seq analysis could be carried out and compare with our present data.

NLRP3 inflammasome consists of a complex of proteins, including NLRP3/ASC/caspase-1, which are responsible for the cleavage of pro-IL- $1 \beta$ into active IL- $1 \beta$ $[50,51]$. IL-1 $\beta$ has been well known as a potent pain 

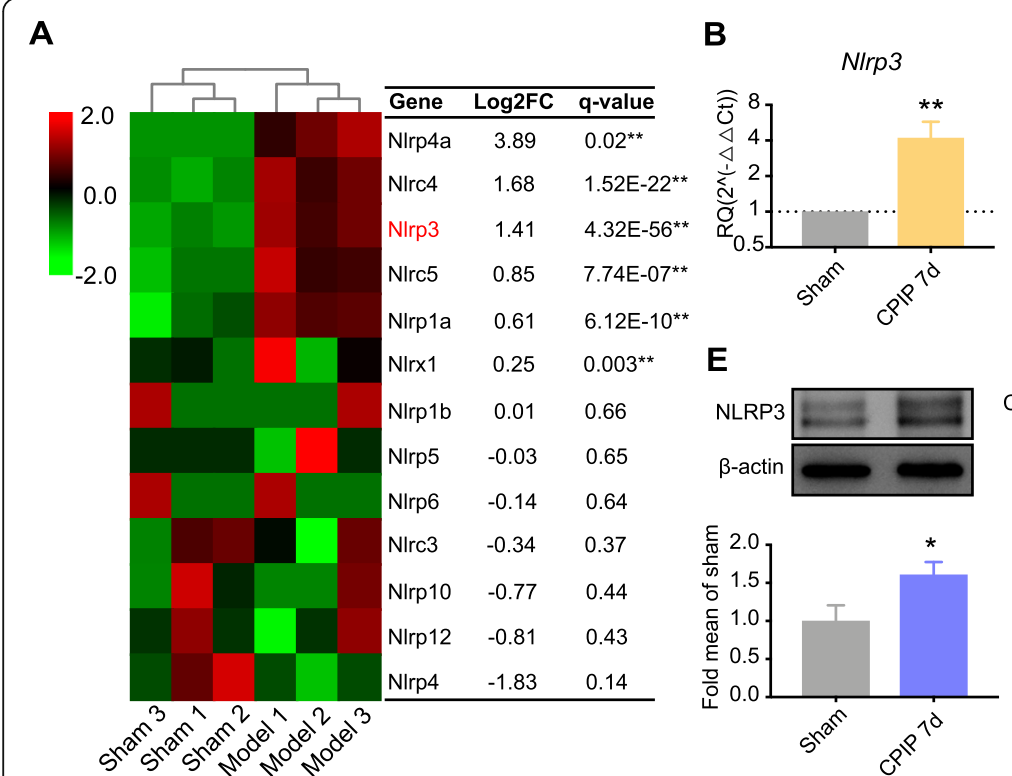

C

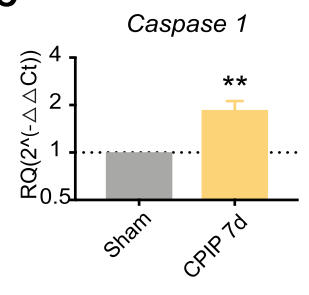

F
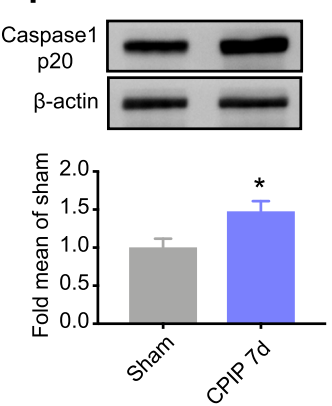

D

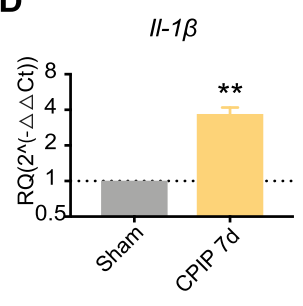

G
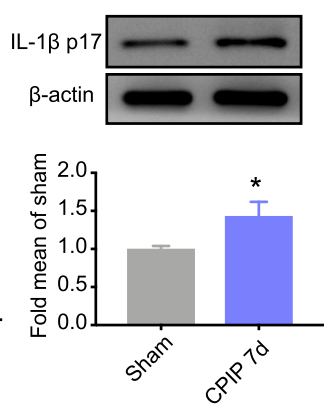

Fig. 8 NLRP3 inflammasome is identified to be significantly upregulated in ipsilateral SCDH of CPIP rats. a Heat map showing the expression of NLR family genes identified in ipsilateral SCDH of CPIP rats vs. sham rats. $n=3$ rats/group. $\mathbf{b}-\mathbf{d}$ qPCR validation of the upregulation of N/rp3 (b), Caspase-1 $(\mathbf{c})$, and $I-1 \beta$ (d) genes in ipsilateral SCDH of CPIP rats vs. sham rats. $n=8$ rats/group. e- $\mathbf{g}$ Western blot analysis of NLRP3 (e), Caspase-1 (f), and IL-1 $\beta$ (g) protein expressions in ipsilateral SCDH of CPIP rats vs. sham rats. $n=8$ rats/group. ${ }^{*} p<0.05$, ${ }^{* *} p<0.01$ vs. sham group. Student's $t$ test was used for comparisons

mediator which sensitizes or directly activates the nociceptors [52]. It has been reported that peripheral NLRP3 inflammasome activation in the peripheral sensory nerves or tissues contributes to paclitaxel-induced neuropathic pain or inflammatory pain [34, 35]. However, the exact contribution of NLRP3 inflammasome in the SCDH to pain mechanisms is still not fully understood yet. Here, we successfully identified the genes encoding the NLRP3 inflammasome complex, including Nlrp3, Caspase-1, and $I l-1 \beta$, to be significantly upregulated in SCDH of CPIP rats by RNA-Seq and qPCR. Western blot further validated our results, showing that the protein expression of NLRP3, Caspase-1, and IL-1 $\beta$ are all upregulated in SCDH of CPIP rats vs. sham rats. IL-1 $\beta$ is a well-established pro-inflammatory cytokine that can activate astrocytes and microglia in SCDH to produce mechanical allodynia $[53,54]$. More recently, it was demonstrated that blocking IL- $1 \beta$ with neutralizing antibody prevented glial activation in SCDH and reduced pain response in a mouse CRPS-I model, suggesting an important role of IL- $1 \beta$ in the pathogenesis of CRPS-I [33]. We found that pharmacological blocking NLRP3 inflammasome activation by intrathecal MCC950 significantly reduced IL-1 $\beta$ overproduction in $\mathrm{SCDH}$ of CPIP rats. This indicates NLRP3 inflammasome is the predominant molecular source for IL-1 $\beta$ overproduction in CPIP condition. MCC950 treatment further attenuated activation of astrocytes and microglia in SCDH of CPIP rats and reduced the mechanical allodynia. Therefore, these results suggest that NLRP3 inflammasome may contribute to the mechanical allodynia of CPIP rats via mechanisms possibly involving IL$1 \beta$ overproduction and activation of astrocytes and microglial in SCDH.

At present, male rats were used exclusively for CPIP model establishment in accordance with previous studies [9]. But epidemiological studies indicated that CRPS-I occurs more frequently in female than in male patients $[55,56]$. This clinical finding is further supported by the observation that female animals showed more nocifensive responses than male animals of the CPIP model [57]. Therefore, it would be important to include both male and female animals in the CPIP model for future studies. This will be important for studying the translational significance of this CRPS-I animal model.

CRPS-I remains an enigmatic symptom that usually initiates after limb injury, fracture, or surgery [58]. The CPIP rat model we used in the present study mimics local tissue ischemia, which could result from limb injury, fracture, or surgery, to recapitulate human CRPS-I. Although it well recapitulates many clinical manifestations of CRPS-I in humans, the CPIP rat model still has its own limitation that it does not mimic the typical clinical factors that may trigger CRPS-I, such as limb injury, 
A

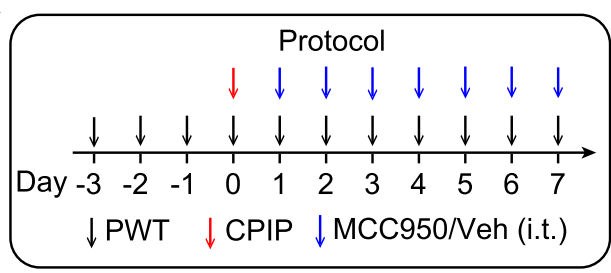

B
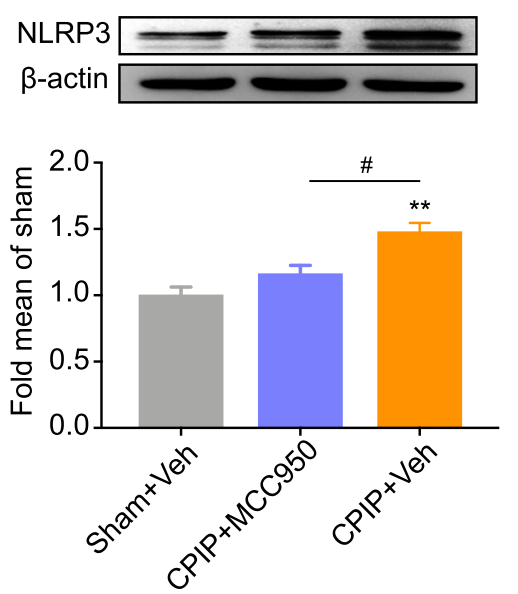

D

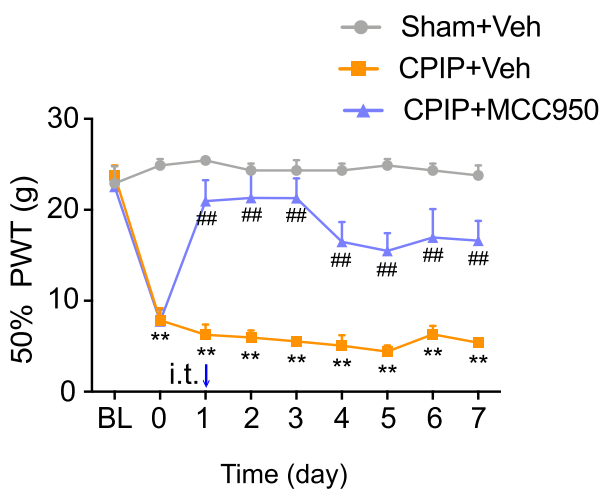

C
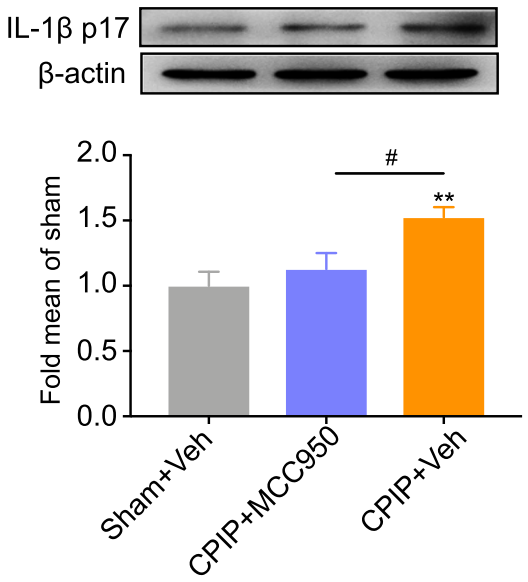

E

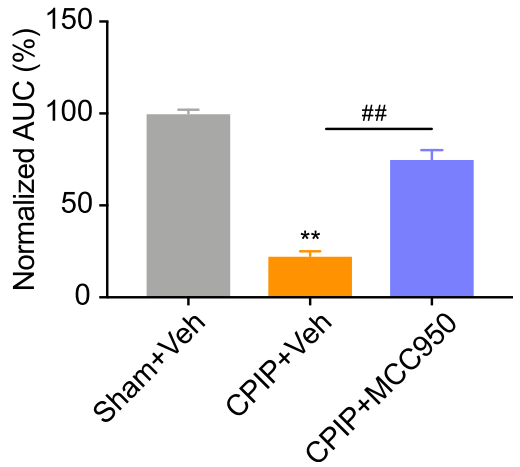

Fig. 9 Pharmacological blocking NLRP3 inflammasome activation attenuated the mechanical allodynia of CPIP rats. a Schematic protocol illustrating the time points for model establishment, behavioral tests, and MCC950 (30 $\mu \mathrm{g} / \mathrm{rat}$ in $12.5 \mu \mathrm{l}$ injection volume, via intrathecal catheter)/vehicle (0.1\% DMSO in PBS) application. b NLRP3 expression in ipsilateral SCDH of Sham+Veh, CPIP+MCC950, and CPIP+Veh groups measure by Western blot. Upper panel indicates representative images of NLRP3 and $\beta$-actin protein expression. Lower panel indicates summarized NLRP3 expression normalized to $\beta$-actin. $\mathbf{c} \|$ L-1 $\beta$ expression in ipsilateral SCDH of Sham+Veh, CPIP+MCC950, and CPIP+Veh groups measure by Western blot. Upper panel indicates representative images of IL-1 13 and $\beta$ actin protein expression. Lower panel indicates summarized IL-1 $\beta$ expression normalized to $\beta$-actin. $n=4$ rats/group. $\mathbf{d}$ Time course effect of MCC950 on $50 \%$ paw withdraw threshold (PWT) of ipsilateral hind paw of CPIP rats. e Summary of the normalized area under the curve (AUC) as in $\mathbf{d} . n=6$ rats/group. ${ }^{* *} p<$ 0.01 vs. Sham+Veh group. ${ }^{\# \prime} p<0.01$ vs. CPIP+Veh group. Two-way ANOVA followed by Tukey's post hoc test was used for comparison in panel d. One-way ANOVA followed by Tukey's post hoc test was used for comparison in panel e

fracture, or surgery. More recently, it is proposed that CRPS is a post-traumatic autoimmune disease [58]. Therefore, some other animal models, such as the mouse tibia fracture model and the newly developed translational passive transfer trauma mouse model of CRPS may be used for further validation of our current findings $[33,59,60]$.

\section{Conclusions}

In conclusion, we presented the gene expression profiling data of ipsilateral SCDH of a rat model of CRPS-I using RNA-Seq. We uncovered a number of DEGs and pathways in the SCDH that may potentially participate in mediating the neuroinflammation and pain of CRPS-I. We further identified NLRP3 inflammasome in SCDH as 

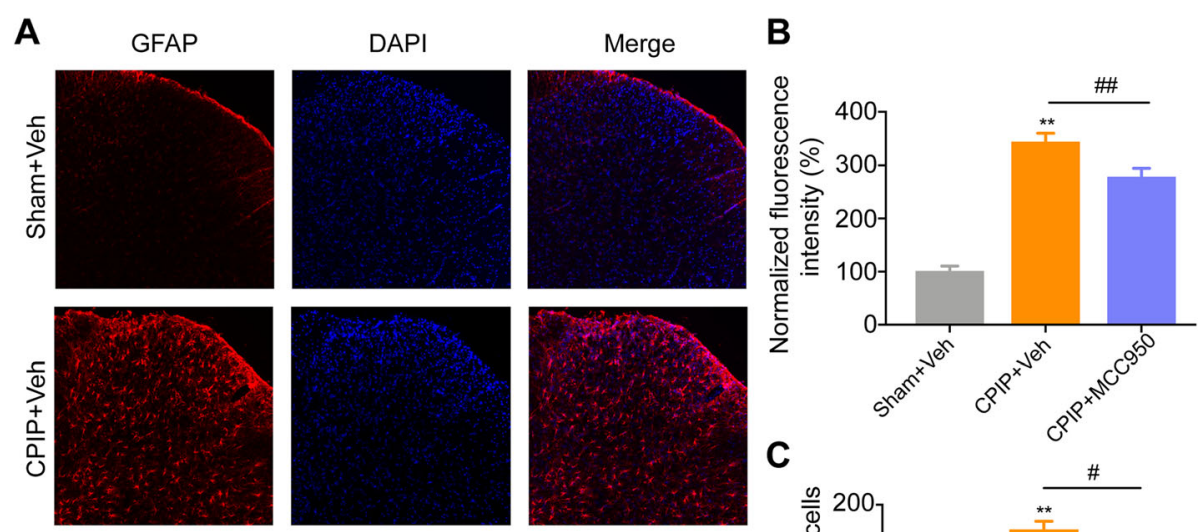

C
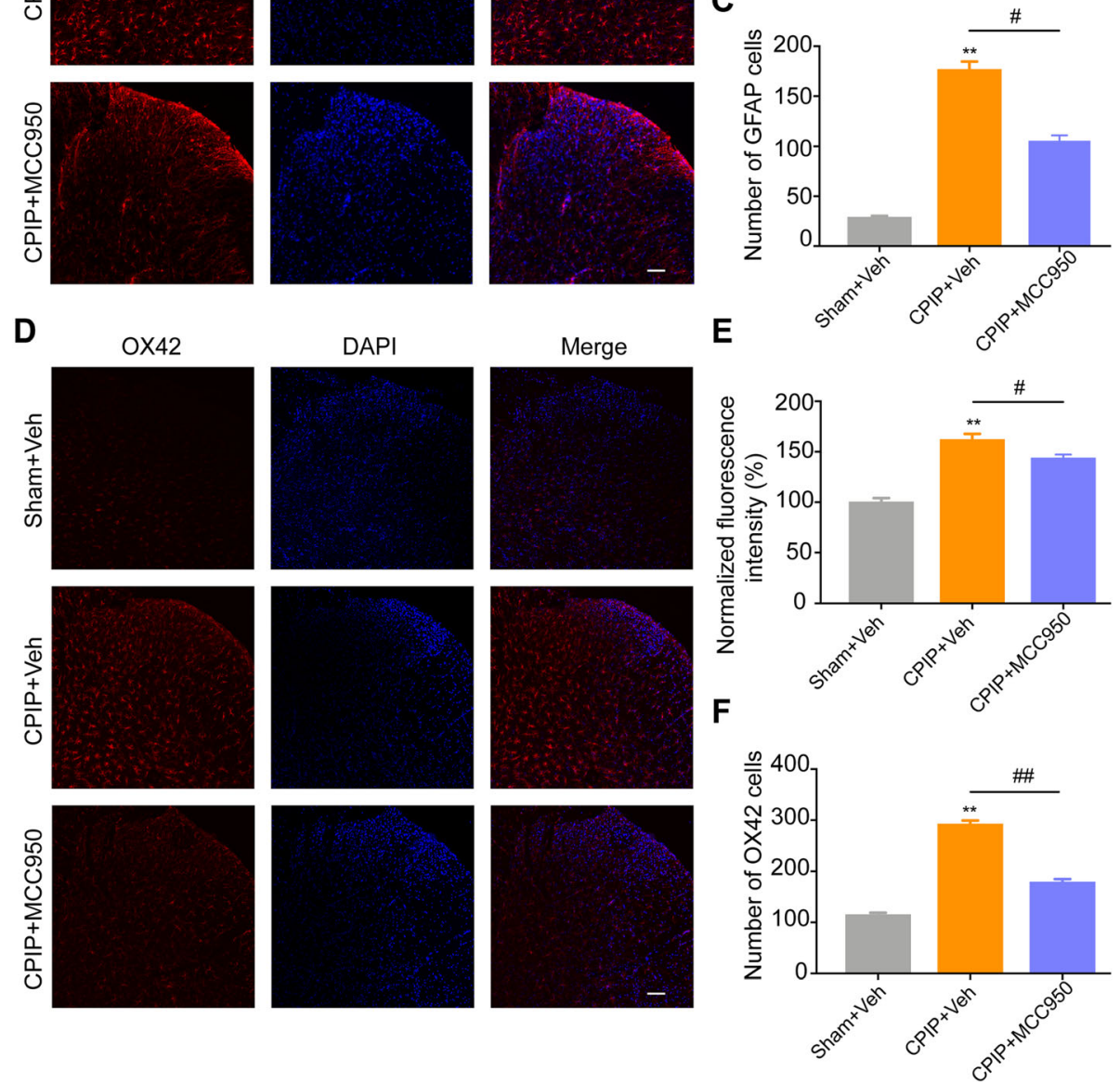

Fig. 10 Pharmacological blocking NLRP3 inflammasome activation attenuates astrocyte and microglial activation in SCDH of CPIP rats. a Representative photos of ipsilateral SCDH stained with GFAP antibody showing astrocytes from Sham+Veh, CPIP+Veh, and CPIP+MCC950 group. Scale bar $=100 \mu \mathrm{m}$. b Summary of the normalized fluorescence intensity (\%) of GFAP staining in ipsilateral SCDH. $n=5$ rats/group. $\mathbf{c}$ Summary of the number of astrocytes observed in ipsilateral SCDH per observation field. $n=5$ rats/group. $\mathbf{d}$ Representative photos of ipsilateral SCDH stained with OX42 antibody showing microglia from Sham+Veh, CPIP+Veh, and CPIP+MCC950 group. Scale bar $=100 \mu$ m. e Summary of the normalized fluorescence intensity (\%) of OX42 staining in ipsilateral SCDH. $n=6$ rats/group. $\mathbf{f}$ Summary of the number of microglia observed in ipsilateral SCDH per observation field. $n=6$ rats/group. ${ }^{* *} p<0.01$ vs. Sham+Veh group. ${ }^{\#} p<0.05,{ }^{\# \#} p<0.01$ vs. CPIP+Veh group. One-way ANOVA followed by Tukey's post hoc test was used for comparison

a key contributor to the pain and inflammation responses in CPIP rats. These results may provide us insights into understanding the molecular mechanisms of CRPS-I and further help to develop novel and effective therapeutics against CRPS-I.

\section{Supplementary information}

Supplementary information accompanies this paper at https://doi.org/10. 1186/s12974-020-01834-0.

Additional file 1: Suppl. Table 1. All expressed transcripts. 
Additional file 2: Suppl. Table 2. DEGs.

Additional file 3: Suppl. Table 3. BP result of the upregulated DEGs in CPIP

Additional file 4: Suppl. Table 4. BP result of the downregulated DEGS in CPIP.

Additional file 5: Suppl. Table 5. CC result of the upregulated DEGs in CPIP

Additional file 6: Suppl. Table 6. CC result of the downregulated DEGs in CPIP.

Additional file 7: Suppl. Table 7. MF result of the upregulated DEGs in CPIP

Additional file 8: Suppl. Table 8. MF result of the downregulated DEGs in CPIP.

Additional file 9: Suppl. Table 9. KEGG analysis.

Additional file 10: Suppl. Table 10. Overlapping with SNI.

Additional file 11: Suppl. Table 11. Overlapping with CCl.

Additional file 12: Suppl. Fig. 1. Analysis of astrocyte activation, microglia activation and oligodendrocyte differentiation using GSEA. (AC) GSEA analysis of CPIP model RNA-Seq dataset using well-defined gene sets for astrocyte activation, microglia activation and oligodendrocyte differentiation from the Molecular Signatures Database v7.1. (D-F) GSEA analysis of the SNI model dataset (GSE18803) for comparison with the CPIP model.

\section{Abbreviations}

CRPS-I: Complex regional pain syndrome type-l; CPIP: Chronic post-ischemic pain; RNA-Seq: RNA-sequencing; SCDH: Spinal cord dorsal horn;

DEGs: Differentially expressed genes; NSAIDs: Non-steroidal anti-inflammatory drugs; DRG: Dorsal root ganglia; PWT: Paw withdrawal threshold; QC: Quality control; KEGG: Kyoto Encyclopedia of Genes and Genomes; DAVID: Data-base for Annotation, Visualization and Integrated Discovery; qPCR: Real-time quantitative PCR; SNI: Spared nerve injury; CCl: Chronic constriction injury; GEO: Gene Expression Omnibus; STRING: Search Tool for the Retrieval of Interacting Genes; PPI: Protein-protein interaction; BP: Biological processes; CC: Cellular component; MF: Molecular function; ROS: Reactive oxygen species

\section{Acknowledgements}

Not applicable.

\section{Authors' contributions}

B.L. (Boyi Liu) and J.F. designed and supervised this study. R.C., Q.H., X.Z., and Y.L. carried out the experiments. C.Y., B.L. (Boyu Liu), and Y.T. analyzed the data. B.L. (Boyi Liu) prepared the manuscript. All authors reviewed the manuscript.

\section{Funding}

This project is supported by Zhejiang Provincial Natural Science Funds for Distinguished Young Scholars (no. LR17H270001), the National Natural Science Foundation of China (no. 81873365, no. 81603676), research funds from Zhejiang Chinese Medical University (no. Q2019J01, no. 2018ZY37, no. 2018ZY19), and Zhejiang Province Top Discipline of Chinese Medicine (no. ZTK2017A03, no. ZTK2017A04). Contents are solely the responsibility of the authors and do not necessarily represent the views of the funders.

\section{Availability of data and materials}

The key data are contained in the figures, tables, and additional files. The datasets used and/or analyzed during this study can be obtained from the corresponding author on reasonable request.

\section{Ethics approval and consent to participate}

This article does not contain any studies with human participants performed by any of the authors. All experimental procedures were carried out in accordance with the National Institutes of Health Guide for the Care and Use of Laboratory Animals (NIH publications no. 8023, revised 1978) and approved by the Animal Ethics Committee of Zhejiang Chinese Medical University (permission number ZSLL-2017-183).
Consent for publication

Not applicable.

\section{Competing interests}

The authors declare that there are no competing interests.

\section{Author details}

1Department of Neurobiology and Acupuncture Research, The Third Clinical Medical College, Zhejiang Chinese Medical University, Key Laboratory of Acupuncture and Neurology of Zhejiang Province, 548 Binwen Road, Hangzhou 310053, China. ${ }^{2}$ Academy of Chinese Medical Sciences, Zhejiang Chinese Medical University, Hangzhou 310053, China.

Received: 28 May 2019 Accepted: 3 May 2020

Published online: 23 May 2020

\section{References}

1. Shim H, Rose J, Halle S, Shekane P. Complex regional pain syndrome: a narrative review for the practicing clinician. Br J Anaesth. 2019.

2. Yeo J, Jung $\mathrm{H}$, Lee $\mathrm{H}$. Effects of glutathione on mechanical allodynia and central sensitization in chronic postischemic pain rats. Pain Res Manag. 2017;2017:7394626.

3. Ott S, Maihofner C. Signs and symptoms in 1,043 patients with complex regional pain syndrome. J Pain. 2018;19:599-611.

4. Urits I, Shen AH, Jones MR, Viswanath O, Kaye AD. Complex regional pain syndrome, current concepts and treatment options. Curr Pain Headache Rep. 2018;22:10.

5. Hord ED, Oaklander AL. Complex regional pain syndrome: a review of evidence-supported treatment options. Curr Pain Headache Rep. 2003;7: 188-96.

6. Hsu ES. Practical management of complex regional pain syndrome. Am J Ther. 2009;16:147-54.

7. Coderre TJ, Bennett GJ. A hypothesis for the cause of complex regional pain syndrome-type I (reflex sympathetic dystrophy): pain due to deep-tissue microvascular pathology. Pain Med. 2010;11:1224-38.

8. Berthelot JM. Current management of reflex sympathetic dystrophy syndrome (complex regional pain syndrome type I). Jt Bone Spine. 2006;73: 495-9.

9. Coderre TJ, Xanthos DN, Francis L, Bennett GJ. Chronic post-ischemia pain (CPIP): a novel animal model of complex regional pain syndrome-type I (CRPS-l; reflex sympathetic dystrophy) produced by prolonged hindpaw ischemia and reperfusion in the rat. Pain. 2004;112:94-105.

10. Klafke JZ, da Silva MA, Rossato MF, de Prá SD, Rigo FK, Walker Cl, Bochi GV, Moresco RN, Ferreira J, Trevisan G. Acute and chronic nociceptive phases observed in a rat hind paw ischemia/reperfusion model depend on different mechanisms. Pflugers Arch. 2016;468:229-41.

11. Tang $Y$, Liu L, Xu D, Zhang W, Zhang Y, Zhou J, Huang W. Interaction between astrocytic colony stimulating factor and its receptor on microglia mediates central sensitization and behavioral hypersensitivity in chronic post ischemic pain model. Brain Behav Immun. 2018;68:248-60.

12. Kim JH, Kim YC, Nahm FS, Lee PB. The therapeutic effect of vitamin $C$ in an animal model of complex regional pain syndrome produced by prolonged hindpaw ischemia-reperfusion in rats. Int J Med Sci. 2017;14:97-101.

13. Yin C, Hu Q, Liu B, Tai Y, Zheng X, Li Y, Xiang X, Wang P, Liu B. Transcriptome profiling of dorsal root ganglia in a rat model of complex regional pain syndrome type-I reveals potential mechanisms involved in pain. J Pain Res. 2019;12:1201-16.

14. Hu Q, Wang Q, Wang C, Tai Y, Liu B, Shao X, Fang J, Liu B. TRPV1 Channel contributes to the behavioral hypersensitivity in a rat model of complex regional pain syndrome type 1. Front Pharmacol. 2019;10:453.

15. Xu J, Tang Y, Xie M, Bie B, Wu J, Yang H, Foss JF, Yang B, Rosenquist RW, Naguib M. Activation of cannabinoid receptor 2 attenuates mechanical allodynia and neuroinflammatory responses in a chronic post-ischemic pain model of complex regional pain syndrome type I in rats. Eur J Neurosci. 2016:44:3046-55.

16. Luo X, Tai WL, Sun L, Pan Z, Xia Z, Chung SK, Cheung CW. Crosstalk between astrocytic CXCL12 and microglial CXCR4 contributes to the development of neuropathic pain. Mol Pain. 2016;12.

17. Ji RR, Chamessian A, Zhang YQ. Pain regulation by non-neuronal cells and inflammation. Science. 2016;354:572-7. 
18. Hu Q, Zheng X, Chen R, Liu B, Tai Y, Shao X, Fang J, Liu B. Chronic postischemia pain model for complex regional pain syndrome type-l in rats. J Vis Exp. 2020.

19. Chai W, Tai Y, Shao X, Liang Y, Zheng GQ, Wang P, Fang J, Liu B. Electroacupuncture alleviates pain responses and inflammation in a rat model of acute gout arthritis. Evid Based Complement Alternat Med. 2018; 2018:2598975

20. Li Y, Yin C, Li X, Liu B, Wang J, Zheng X, Shao X, Liang Y, Du J, Fang J, Liu B. Electroacupuncture alleviates paclitaxel-induced peripheral neuropathic pain in rats via suppressing TLR4 signaling and TRPV1 upregulation in sensory neurons. Int J Mol Sci. 2019;20.

21. Chaplan SR, Bach FW, Pogrel JW, Chung JM, Yaksh TL. Quantitative assessment of tactile allodynia in the rat paw. J Neurosci Methods. 1994;53: 55-63.

22. Liu B, Tai Y, Achanta S, Kaelberer MM, Caceres Al, Shao X, Fang J, Jordt SE. IL-33/ST2 signaling excites sensory neurons and mediates itch response in a mouse model of poison ivy contact allergy. Proc Natl Acad Sci U S A. 2016; 113:E7572-e7579.

23. Liu B, Tai Y, Caceres Al, Achanta S, Balakrishna S, Shao X, Fang J, Jordt SE. Oxidized phospholipid OxPAPC Activates TRPA1 and contributes to chronic inflammatory pain in mice. PLoS One. 2016;11:e0165200.

24. Chen W, Ding H, Zhou X, Lin H, Chou KC. iRNA(m6A)-PseDNC: Identifying Nmethyladenosine sites using pseudo dinucleotide composition. Anal Biochem. 2018;561-562:59-65

25. Liu B, Tai Y, Liu B, Caceres Al, Yin C, Jordt SE. Transcriptome profiling reveals Th2 bias and identifies endogenous itch mediators in poison ivy contact dermatitis. JCl Insight. 2019:5.

26. Subramanian A, Tamayo P, Mootha VK, Mukherjee S, Ebert BL, Gillette MA, Paulovich A, Pomeroy SL, Golub TR, Lander ES, Mesirov JP. Gene set enrichment analysis: a knowledge-based approach for interpreting genomewide expression profiles. Proc Natl Acad Sci U S A. 2005;102:15545-50

27. Caceres Al, Liu B, Jabba SV, Achanta S, Morris JB, Jordt SE. Transient receptor potential cation channel subfamily $M$ member 8 channels mediate the antiinflammatory effects of eucalyptol. Br J Pharmacol. 2017;174:867-79.

28. Livak KJ, Schmittgen TD. Analysis of relative gene expression data using real-time quantitative PCR and the 2(-Delta Delta C(T)) Method. Methods. 2001;25:402-8.

29. Du H, Shi J, Wang M, An S, Guo X, Wang Z. Analyses of gene expression profiles in the rat dorsal horn of the spinal cord using RNA sequencing in chronic constriction injury rats. J Neuroinflammation. 2018;15:280.

30. Franceschini A, Szklarczyk D, Frankild S, Kuhn M. STRING v9.1: proteinprotein interaction networks, with increased coverage and integration. Nucleic Acids Res. 2013;41:D808-15

31. Shannon P, Markiel A, Ozier O, Baliga NS, Wang JT, Ramage D, Amin N, Schwikowski B, Ideker T. Cytoscape: a software environment for integrated models of biomolecular interaction networks. Genome Res. 2003;13:2498-504.

32. Hu Q, Zheng X, Li X, Liu B, Yin C, Li Y, Chen R, Wang J, Liang Y, Shao X, et al. Electroacupuncture alleviates mechanical allodynia in a rat model of complex regional pain syndrome type-I via suppressing spinal CXCL12/ CXCR4 signaling. J Pain. 2020

33. Helyes Z, Tekus V, Szentes N, Pohoczky K, Botz B, Kiss T, Kemeny A, Kornye $Z$, Toth $K$, Lenart $N$, et al. Transfer of complex regional pain syndrome to mice via human autoantibodies is mediated by interleukin-1-induced mechanisms. Proc Natl Acad Sci U S A. 2019;116:13067-76.

34. Yin C, Liu B, Wang P, Li X, Li Y, Zheng X, Tai Y, Wang C, Liu B. Eucalyptol alleviates inflammation and pain responses in a mouse model of gout arthritis. Br J Pharmacol. 2019.

35. Jia M, Wu C, Gao F, Xiang H, Sun N, Peng P, Li J, Yuan X, Li H, Meng X, et al. Activation of NLRP3 inflammasome in peripheral nerve contributes to paclitaxel-induced neuropathic pain. Mol Pain. 2017;13:1744806917719804

36. Gao F, Xiang HC, Li HP, Jia M, Pan XL, Pan HL, Li M. Electroacupuncture inhibits NLRP3 inflammasome activation through CB2 receptors in inflammatory pain. Brain Behav Immun. 2018;67:91-100.

37. He W, Long T, Pan Q, Zhang S, Zhang Y, Zhang D, Qin G, Chen L, Zhou J. Microglial NLRP3 inflammasome activation mediates IL-1 beta release and contributes to central sensitization in a recurrent nitroglycerin-induced migraine model. J Neuroinflammation. 2019;16:78.

38. Wang Q, Ou Y, Hu G, Wen C, Yue S, Chen C, Xu L, Xie J, Dai H, Xiao H, et al. Naringenin attenuates nonalocholic fatty liver disease by downregulating NLRP3/NF-kappaB pathway in mice. Br J Pharmacol. 2019.
39. Zahid A, Li B, Kombe AJK, Jin T, Tao J. Pharmacological inhibitors of the NLRP3 inflammasome. Front Immunol. 2019;10:2538.

40. Chen G, Zhang YQ, Qadri YJ, Serhan CN, Ji RR. Microglia in pain: detrimental and protective roles in pathogenesis and resolution of pain. Neuron. 2018; 100:1292-311.

41. Ji RR, Donnelly CR, Nedergaard M. Astrocytes in chronic pain and itch. Nat Rev Neurosci. 2019;20:667-85.

42. Ji RR, Nackley A, Huh Y, Terrando N, Maixner W. Neuroinflammation and central sensitization in chronic and widespread pain. Anesthesiology. 2018; 129:343-66

43. Matsuda M, Huh Y, Ji RR. Roles of inflammation, neurogenic inflammation, and neuroinflammation in pain. J Anesth. 2019;33:131-9.

44. Kiguchi N, Kobayashi D, Saika F, Matsuzaki S, Kishioka S. Pharmacologica regulation of neuropathic pain driven by inflammatory macrophages. Int J Mol Sci. 2017:18.

45. Tsuda M, Inoue K. Neuron-microglia interaction by purinergic signaling in neuropathic pain following neurodegeneration. Neuropharmacology. 2016; 104:76-81.

46. Kallenborn-Gerhardt W, Schröder K, Del Turco D, Lu R, Kynast K, Kosowski J, Niederberger E, Shah AM, Brandes RP, Geisslinger G, Schmidtko A. NADPH oxidase-4 maintains neuropathic pain after peripheral nerve injury. J Neurosci. 2012:32:10136-45.

47. Kallenborn-Gerhardt W, Schröder K, Geisslinger G, Schmidtko A. NOXious signaling in pain processing. Pharmacol Ther. 2013:137:309-17.

48. Park JM, Kim CK, Lee HC, Jung H, Choi KU, Hong SW, Lim DG, Baek WY, Kwak KH. Antiallodynic effects of vitamin $\mathrm{C}$ and vitamin $\mathrm{E}$ in chronic postischemia pain rat model. Korean J Anesthesiol. 2013;65:442-8.

49. Kwak KH, Han CG, Lee SH, Jeon Y, Park SS, Kim SO, Baek WY, Hong JG, Lim DG. Reactive oxygen species in rats with chronic post-ischemia pain. Acta Anaesthesiol Scand. 2009;53:648-56.

50. Swanson KV, Deng M, Ting JP. The NLRP3 inflammasome: molecular activation and regulation to therapeutics. Nat Rev Immunol. 2019;19:477-89.

51. Alexander SPH, Fabbro D, Kelly E, Mathie A, Peters JA, Veale EL, Armstrong JF, Faccenda E, Harding SD, Pawson AJ, et al. The concise guide to pharmacology 2019/20: catalytic receptors. Br J Pharmacol. 2019;176(Suppl 1):S247-96.

52. Binshtok AM, Wang $H$, Zimmermann $K$, Amaya $F$, Vardeh $D$, Shi L, Brenner GJ, Ji RR, Bean BP, Woolf CJ, Samad TA. Nociceptors are interleukin-1 beta sensors. J Neurosci. 2008;28:14062-73.

53. Yan X, Li F, Maixner DW, Yadav R, Gao M, Ali MW, Hooks SB, Weng HR. Interleukin-1beta released by microglia initiates the enhanced glutamatergic activity in the spinal dorsal horn during paclitaxel-associated acute pain syndrome. Glia. 2019;67:482-97.

54. Wei XH, Yang T, Wu Q, Xin WJ, Wu JL, Wang YQ, Zang Y, Wang J, Li YY, Liu $X G$. Peri-sciatic administration of recombinant rat IL-1beta induces mechanical allodynia by activation of src-family kinases in spinal microglia in rats. Exp Neurol. 2012;234:389-97.

55. Weissmann R, Uziel Y. Pediatric complex regional pain syndrome: a review. Pediatr Rheumatol Online J. 2016;14:29.

56. Kim H, Lee $\mathrm{CH}$, Kim SH, Kim YD. Epidemiology of complex regional pain syndrome in Korea: an electronic population health data study. PLoS One. 2018;13:e0198147.

57. Tang C, Li J, Tai WL, Yao W, Zhao B, Hong J, Shi S, Wang S, Xia Z. Sex differences in complex regional pain syndrome type I (CRPS-I) in mice. J Pain Res. 2017;10:1811-9.

58. Cuhadar U, Gentry C, Vastani N, Sensi S, Bevan S, Goebel A, Andersson DA Autoantibodies produce pain in complex regional pain syndrome by sensitizing nociceptors. Pain. 2019;160:2855-65.

59. Li WW, Guo TZ, Shi X, Birklein F, Schlereth T, Kingery WS, Clark JD. Neuropeptide regulation of adaptive immunity in the tibia fracture model of complex regional pain syndrome. J Neuroinflammation. 2018;15:105.

60. Birklein F, Ibrahim A, Schlereth T, Kingery WS. The rodent tibia fracture model: a critical review and comparison with the complex regional pain syndrome literature. J Pain. 2018;19:1102 e1101-19.

\section{Publisher's Note}

Springer Nature remains neutral with regard to jurisdictional claims in published maps and institutional affiliations. 